\title{
Modified Prüfer and EFGP Transforms and the Spectral Analysis of One-Dimensional Schrödinger Operators
}

\author{
Alexander Kiselev ${ }^{1, \star}$, Yoram Last ${ }^{2}$, Barry Simon ${ }^{2, \star \star}$ \\ 1 Department of Mathematics, University of Chicago, Chicago, IL 60637, USA \\ 2 Division of Physics, Mathematics, and Astronomy, California Institute of Technology 253-37, Pasadena, \\ CA 91125 , USA
}

Received: 8 April 1997 / Accepted: 19 June 1997

\begin{abstract}
Using control of the growth of the transfer matrices, we discuss the spectral analysis of continuum and discrete half-line Schrödinger operators with slowly decaying potentials. Among our results we show if $V(x)=\sum_{n=1}^{\infty} a_{n} W\left(x-x_{n}\right)$, where $W$ has compact support and $x_{n} / x_{n+1} \rightarrow 0$, then $H$ has purely a.c. (resp. purely s.c.) spectrum on $(0, \infty)$ if $\sum a_{n}^{2}<\infty$ (resp. $\left.\sum a_{n}^{2}=\infty\right)$. For $\lambda n^{-1 / 2} a_{n}$ potentials, where $a_{n}$ are independent, identically distributed random variables with $E\left(a_{n}\right)=0, E\left(a_{n}^{2}\right)=1$, and $\lambda<2$, we find singular continuous spectrum with explicitly computable fractional Hausdorff dimension.
\end{abstract}

\section{Introduction}

In this paper, we will study continuum and discrete Schrödinger operators on the halfline (while we don't always make them explicit, given theory in [10, 26, 32], many of our results extend to suitable whole-line problems). Explicitly, we are interested in the spectral analysis of operators $H$ on $L^{2}(0, \infty ; d x)$ and on $\ell^{2}([1, \infty))$ given by

$$
(H u)(x)=-\frac{d^{2}}{d x^{2}}+V(x)
$$

in the continuum case and

$$
(H u)(n)=u(n+1)+u(n-1)+V(n) u(n)
$$

in the discrete case.

\footnotetext{
* Research supported in part by NSF Grant No. DMS-9022140.

$\star \star$ This material is based upon work supported by the National Science Foundation under Grant No. DMS9401491. The Government has certain rights in this material.
} 
Suitable boundary conditions are set at $x$ (or $n)=0$, so that $H$ is self-adjoint (since in all our examples $V$ is limit point at infinity, a boundary condition is not needed there). We are interested in the spectral properties of such operators in situations where $|V(n)| \rightarrow 0$ as $n \rightarrow \infty$, but so slowly that the usual scattering theory will not apply. Our main theme in this paper is that there are perturbation techniques of remarkable power for such operators based on two ideas.

The first is that one can use the transfer matrix to analyze spectral properties. The transfer or fundamental matrix is a $2 \times 2$ unimodular matrix defined in the continuum case for any $E$ by

$$
T_{E}(x, 0)\left(\begin{array}{l}
a \\
b
\end{array}\right)=\left(\begin{array}{l}
u^{\prime}(x) \\
u(x)
\end{array}\right),
$$

where $-u^{\prime \prime}+V u=E u, u^{\prime}(0)=a, u(0)=b$. In the discrete case

$$
T_{E}(n, 0)\left(\begin{array}{l}
a \\
b
\end{array}\right)=\left(\begin{array}{c}
u(n+1) \\
u(n)
\end{array}\right),
$$

where $u(1)=a, u(0)=b$, and $u(n+1)+u(n-1)+V(n) u(n)=E u(n)$.

The second idea is that one can control the transfer matrix by controlling the norms of two solutions of $-u^{\prime \prime}+V u=E u$ and that the critical equations are ones that involve those norms.

Two of us [22] have recently found several new criteria for singular or absolutely continuous spectra in terms of transfer matrices, and these criteria will make some of our results here possible. The perturbation equations we will use have not been systematically used in this context except in the paper of Kotani-Ushiroya [21] whose techniques have some overlap with this paper. But they didn't control the discrete case and their method is so entwined with certain Martingale inequalities that it is unclear how to use them in other contexts.

While we were writing up the work for this paper, we received a preprint from Remling [29] that uses this two-pronged approach and has considerable overlap with our Sects. 5 and 6. We will discuss the connection shortly.

Here are some of the theorems that we will use that relate spectral properties to behavior of $T(n)$. The first is from [22]:

Theorem 1.1. Suppose that there is a fixed sequence $n_{i} \rightarrow \infty$ and $S$ is a subset of $\mathbb{R}$ so that for a.e. $E \in S, \lim _{i \rightarrow \infty}\left\|T_{E}\left(n_{i}, 0\right)\right\|=\infty$. Then $\mu_{\mathrm{ac}}(S)=0$, where $\mu_{\mathrm{ac}}$ is the absolutely continuous part of the spectral measure for $H$.

Remarks. 1. The interesting aspect of this theorem is that $n_{i}$ is arbitrary. The result actually allows a more general sequence $\left\|T_{E}\left(n_{i}, m_{i}\right)\right\|$.

2. In typical applications, $S$ is an interval in the essential spectrum.

In the other direction, one has the following pair of results:

Theorem 1.2. Suppose $S$ is a set so that for a.e. $E \in S, \overline{\lim }_{x \rightarrow \infty}\left\|T_{E}(x, 0)\right\|<\infty$. Then $\mu_{\mathrm{ac}}(Q)>0$ for any $Q \subset S$ with $|Q|>0$ where $|\cdot|=$ Lebesgue measure.

Theorem 1.3. Suppose there is a sequence $n_{i} \rightarrow \infty$ so that $\int_{a}^{b}\left\|T_{E}\left(n_{i}, 0\right)\right\|^{4} d E<\infty$. Then $(a, b) \subset \operatorname{spec}(H)$ and the spectral measure is purely absolutely continuous on $(a, b)$ and $\mu_{\mathrm{ac}}(Q)>0$ for any $Q$ with $|Q \cap(a, b)|>0$. 
Remarks. 1. That Theorem 1.2 is implied by the Gilbert-Pearson [11] theory was noted by Stolz [36]. A simple proof can be found in [33]. Last-Simon [22] prove a stronger variant in which $\left\|T_{E}(x, 0)\right\|$ is replaced by $\int_{-1}^{1}\left\|T_{E}(x+y, 0)\right\| d y$ and $\varlimsup$ by $\underline{\lim }$. (The discrete analog holds with $\underline{\lim }$ and without any local integration.)

2. Theorem 1.3 is from Last-Simon [22] although the method used there is not much more than what is in Carmona [1].

As to distinguishing dense pure point from singular continuous spectrum, in one direction we have the following elementary result from Simon-Stolz [35].

Theorem 1.4. If $\sum_{n}\left\|T_{E}(n, 0)\right\|^{-2}=\infty$ in the discrete case or $\int_{0}^{\infty}\left\|T_{E}(x, 0)\right\|^{-2} d x=$ $\infty$ in the continuum case, then $H u=E u$ has no solution which is $L^{2}$ at infinity.

The paradigm of results that guarantees a solution $L^{2}$ at $\infty$ is Ruelle's proof [30] of Osceledec's theorem. His argument is abstracted in [22]. We will need the following in Sect. 8:

Theorem 1.5. If $\lim _{n \rightarrow \infty}\left[\log \left\|T_{E}(n, 0)\right\| / n^{\alpha}\right]$ exists and lies in $(0, \infty)$ for some $\alpha>0$, then $H u=E u$ has an $L^{2}$ solution.

[22] also has a general abstract result on power decay which, to get an $L^{2}$ solution, requires

$$
\lim _{n \rightarrow \infty} \frac{\log \left\|T_{E}(n, 0)\right\|}{\log n}>\frac{3}{2} .
$$

[22] also has an example where the limit is $\frac{3}{2}$ and there is no $\ell^{2}$ solution. But there are stronger results that hold a.e. in certain probabilistic situations, so we won't discuss the power decay result here. In Sect. 8, we will discuss the probabilistic result.

As for the technique to control the growth of solutions, in the continuum case we will use modified Prüfer variables defined for $E>0$ by

$$
\begin{aligned}
u^{\prime}(x) & =\sqrt{E} R(x) \cos (\theta(x)), \\
u(x) & =R(x) \sin (\theta(x)) .
\end{aligned}
$$

One finds these obey the differential equations (with $k=\sqrt{E}$ )

$$
\begin{gathered}
\frac{d \theta}{d x}=k-\frac{V(x)}{k} \sin ^{2}(\theta(x)), \\
\frac{d \log R(x)}{d x}=\frac{1}{2 k} V(x) \sin (2 \theta(x)) .
\end{gathered}
$$

Two features of these equations are immediately noteworthy:

(a) They separate in the sense that (1.6) does not involve $R$ and after solving it, one obtains $R$ by integration. That $R$ drops out of (1.6) and the right side of (1.7) is an expression of the linearity of the initial equations.

(b) If $V=0$ in some region $(a, b)$, then in that region $R$ is constant and $\theta(x)=\theta(a)+$ $k(x-a)$. It is this fact that leads one to take the factor $\sqrt{E}$ in $(1.5 \mathrm{a})$. The addition of this $\sqrt{E}$ is what distinguishes this from ordinary Prüfer transformations. 
There is a third significant feature which we will turn to momentarily.

Given how common these continuum equations are, we would have expected their discrete analogs would have been rediscovered and used many times, but even after some efforts at tracking various literature, we've found them in a single chain of four papers (and we are a fifth in this chain, since we learned of them from the fourth paper!).

The original discoverer of the correct equation was Thomas Eggarter [9] in 1971. He was not looking at an explicit difference equation but rather a continuum equation with $V(x)=V_{0} \sum_{i=1}^{n} \delta\left(x-x_{i}\right)$. By integrating modified Prüfer variables across the $\delta$-functions, he was led to the transforms $(E=2 \cos (k))$,

$$
\begin{aligned}
u(n)-\cos (k) u(n-1) & =R(n) \cos (\theta(n)), \\
\sin (k) u(n-1) & =R(n) \sin (\theta(n)),
\end{aligned}
$$

in which case we have, after some calculation (see Sect. 2),

$$
\begin{gathered}
\cot (\theta(n+1))=\cot (k+\theta(n))-(\sin (k))^{-1} V(n), \\
\frac{R(n+1)^{2}}{R(n)^{2}}=1-\frac{V(n)}{\sin (k)} \sin (2 \theta(n)+2 k)+\frac{V(n)^{2}}{\sin ^{2}(k)} \sin ^{2}(\theta(n)+k) .
\end{gathered}
$$

Actually, he had only an equation of the form (1.9). The definition of $\theta(n)$ and precise (1.9) is in a 1975 paper of Gredeskul-Pastur [13] who followed up on Eggarter's work.

$[9,13]$ focus on (1.9) because they use the transform to study the integrated density of states. Pastur-Figotin [26] defined $R$ and exploited (1.10) to study the Lyapunov exponent. In recognition of these seminal works, we call (1.8) the EFGP transform. Their approach was further exploited in Chulaevsky-Spencer [2]. It will often be useful to use an equivalent form of (1.9) that appears as (2.14).

Notice that (1.9), (1.10) have the two critical properties (a), (b) mentioned for (1.6), (1.7) in the continuum case. In particular, if $V(n)=0$ for $n$ in some interval $\left[n_{0}, n_{1}\right]$, then in that region $R(n)$ is constant and

$$
\theta(n)=\theta\left(n_{0}\right)+k\left(n-n_{0}\right) .
$$

While the EFGP transform was obtained by integrating a continuum $\delta$-function model, it could also be found by looking for a transform with property (b). We will explain this in Sect. 2.

$[9,13,26,2]$ all consider $V$ 's with no decay as $n \rightarrow \infty$ but with a small coupling so that any calculations are only asymptotic in coupling constant. It turns out that the methods are especially well suited when $V(n) \rightarrow 0$ at infinity and one obtains results that are exact for a fixed $V$. For example, in Sect. 8, we will find exact formulas for the local Hausdorff dimensions of certain singular continuous spectral measures.

The third critical factor of the modified Prüfer and EFGP transforms is a major theme of this paper, namely, that first-order terms in $V$ are oscillatory while the second-order term has a strong tendency to be strictly positive. This idea is already seen in $[26,2]$, where $\gamma(E)$ is $O\left(g^{2}\right)$ with $g$ a coupling constant because the first-order terms drop out.

Let us be explicit about this idea. In (1.6), one might think the positivity comes via the square in $\sin ^{2}(\theta(x))$ but that is wrong! Indeed, in writing $\sin ^{2}(\theta)=\frac{1}{2}-\frac{1}{2} \cos (2 \theta)$, it is the $\cos (2 \theta)$ that is critical! Formally, (1.6) says

$$
\theta(x)=k x+\theta_{0}-\frac{V(x)}{k} \sin ^{2}\left(k x+\theta_{0}\right)+O\left(V^{2}\right) \equiv k x+\theta_{0}+\delta \theta+O\left(V^{2}\right),
$$


and then using

$$
\sin (2 \theta)=\sin \left(2 k x+2 \theta_{0}\right)+2 \cos \left(2 k x+2 \theta_{0}\right) \delta \theta+O\left(V^{2}\right),
$$

we get

$$
\frac{d \log R}{d x}=t_{1}+t_{2}+O\left(V^{3}\right)
$$

where

$$
t_{1}=\frac{1}{2 k} V(x) \sin \left(2\left(k x+\theta_{0}\right)\right)-\frac{1}{2 k^{2}}\left(V(x) \int_{x_{0}}^{x} V(y) d y\right) \cos \left(2\left(k x+\theta_{0}\right)\right)
$$

is the oscillatory term that is often unimportant, while

$$
t_{2}=\frac{1}{4 k^{2}} \frac{d}{d x}\left[\int_{x_{0}}^{x} V(y) \cos \left(2 k y+2 \theta_{0}\right) d y\right]^{2}
$$

has a positive integral, second order in $V$.

In explicit cases, it is more subtle to prove the second order is strictly positive and, indeed, for examples like $V(x)=x^{-\alpha}, \alpha<\frac{1}{2}$, where the spectrum is absolutely continuous (by Weidmann [37]), the second-order terms do not cause divergences. This means that results that depend on a finite second-order term should hold more generally than those that depend on an infinite second-order term. Indeed, we

Conjecture. If $V$ is bounded and in $L^{2}(\mathbb{R}, d x)$ (or $\left.\ell^{2}\left(\mathbb{Z}_{+}\right)\right)$, then the essential support of the a.c. part of the spectrum is all of $(0, \infty)$ (or $(-2,2)$ in the discrete case).

Our idea is that for almost all (but not all; see, e.g., [24, 25, 34]) $k$, the oscillations should kill the first-order term, and so the $L^{2}$ condition should suffice to give a bounded transfer matrix for a.e. $k$ and so the stated conclusion about the a.c. spectrum by Theorem 1.2.

After discussing the modified Prüfer and EFGP transforms and their relation to the growth of the transfer matrix in Sect. 2, we turn to two warm-up problems in Sects. 3 and 4. In Sect. 3, we show these transforms can replace the Harris-Lutz [15] method in many cases where that method is applicable. In Sect. 4, we look at potentials $V$ with $\lim x|V(x)|$ finite and show that for such potentials their positive eigenvalues can only coalesce at $E=0$. Since examples are known with countable many eigenvalues embedded in $(0, \infty)$, this result is interesting.

In Sects. 5-7, we study sparse potentials.

Definition. A Pearson potential is one of the form

$$
V(x)=\sum_{n=1}^{\infty} a_{n} W\left(x-x_{n}\right),
$$

where $W$ is a bounded, non-negative function of compact support, $a_{n} \rightarrow 0$, and $1 \leq$ $x_{1}<x_{2}<x_{3}<\cdots$,

$$
\frac{x_{n}}{x_{n+1}} \rightarrow 0
$$


The name is in honor of David Pearson who considered potentials of the form (1.11) where $\sum_{n=1}^{\infty} a_{n}^{2}=\infty$ and $x_{n}$ went to infinity sufficiently fast. To make things precise, think of the example $x_{n}=n$ !.

Our major goal in Sects. 5-6 is to prove the following:

\section{Theorem 1.6. Let $V$ be a Pearson potential. Then}

(1) If $\sum_{n=1}^{\infty} a_{n}^{2}<\infty$, the spectrum of $-\frac{d^{2}}{d x^{2}}+V(x)$ is purely absolutely continuous on $(0, \infty)$ for any boundary condition at 0 .

(2) If $\sum_{n=1}^{\infty} a_{n}^{2}=\infty$, the spectrum of $-\frac{d^{2}}{d x^{2}}+V(x)$ is purely singular continuous on $(0, \infty)$ for any boundary condition at 0 .

In Sect. 5, we will actually prove a stronger version of (1):

Theorem 1.6'. Let $V$ have the form (1.11) where

$$
\varlimsup \frac{x_{n}}{x_{n+1}}<1
$$

Then (1) holds.

Pearson [27, 28] proved a weak version of (2) in that if $\sum_{n=1}^{\infty} a_{n}^{2}=\infty$, there exists some set of $x_{n}$ 's so that the spectrum is purely singular continuous. In [27], there are hints that a result of type (1) (again with $x_{n}$ sufficiently large) should hold, but nothing explicit.

As noted at the end of Sect. 5, for (1) the bumps $W\left(x-x_{n}\right)$ can be $n$-dependent.

At the end of Sect. 6 , for $[a, b] \equiv S \subset(0, \infty)$, we construct Pearson-like potentials (bumps whose width grows with $n$ ) so that there is purely a.c. spectrum on $S$ and purely s.c. spectrum on $(0, \infty) \backslash S$.

In a recent paper, coincident with our work, Remling [29] obtained results related to Theorem 1.6(1) using similar methods. He only obtains the existence of absolutely continuous spectrum (his results are consistent with simultaneous singular continuous spectrum while we prove there is none), and he needs at least $\exp \left(\frac{3}{2} n \log n\right)$ growth on the $x_{n}$ (whereas, if $f(n)$ is a monotone function with $f(m) \rightarrow \infty$ no matter how slowly, then $x_{n}=\exp (n f(n))$ obeys (1.12) and $x_{n}=\exp ($ an $)$ obeys (1.13)).

After this manuscript was completed, we obtained a preliminary version of a preprint of Molchanov [23] with considerable overlap with our results in Sects. 5 and 6.

In Sect. 7, we will prove

Theorem 1.7. Let $x_{n} \in \mathbb{Z}$ obey $x_{n} / x_{n+1} \rightarrow 0$. Let $V$ be the potential with

$$
\begin{aligned}
V\left(x_{n}\right) & =a_{n}, \\
V(x) & =0 \quad x \neq x_{n} \text { for any } n .
\end{aligned}
$$

Then,

(1) If $\sum a_{n}^{2}<\infty$, the discrete Schrödinger operator with potential $V$ has purely a.c. spectrum for $(-2,2)$.

(2) If $\sum a_{n}^{2}=\infty$, the operator has purely singular continuous spectrum on $(-2,2)$. 
In Sects. 8 and 9, we discuss models with randomness and decay, first studied by Simon [31] and then by Delyon, et al. [7], Delyon [6], and Kotani-Ushiroya [21]. Typical of the models discussed in these sections is ( $g$ is positive constant)

$$
V(n)=g n^{-\alpha} a_{n},
$$

where the $a_{n}$ are independent, identically distributed random variables, uniformly distributed in $[-1,1]$. We prove

(i) If $\alpha>\frac{1}{2}$, the spectrum is almost surely purely absolutely continuous in $(-2,2)$.

(ii) If $0<\alpha<\frac{1}{2}$, the spectrum is almost surely dense pure point in $(-2,2)$.

(iii) If $\alpha=\frac{1}{2}$, the spectrum is almost surely purely singular continuous in the region $|E|<\left(4-\frac{1}{3} g^{2}\right)^{1 / 2}$ and dense pure point in the region $\left(4-\frac{1}{3} g^{2}\right)^{1 / 2} \leq|E|<2$ (if $g^{2}>12$, interpret $\left(4-\frac{1}{3} g^{2}\right)^{1 / 2}$ as 0$)$.

(iv) In case $\alpha=\frac{1}{2}$ and $g^{2}<12$, in the region $|E|<\left(4-\frac{1}{3} g^{2}\right)^{1 / 2}$, the spectrum has fractional Hausdorff dimension with local dimension $\left(4-E^{2}-\frac{g^{2}}{3}\right) /\left(4-E^{2}\right)$.

Section 8 handles the discrete case, and Sect. 9 the continuum case.

For sparse potentials, we give the details in the continuum case and sketch the discrete case; while for random decaying potentials, we give details in the discrete case and sketch the continuum case.

A.K. would like to thank the hospitality of I.H.E.S., and B.S. the hospitality of Hebrew University where some of this work was done.

\section{Modified Prüfer and EFGP Transforms}

We will be interested in solutions of

$$
-u^{\prime \prime}(x)+V(x) u(x)=k^{2} u(x) .
$$

Change variables to

$$
\begin{aligned}
u^{\prime}(x) & =k R(x) \cos (\theta(x)), \\
u(x) & =R(x) \sin (\theta(x)) .
\end{aligned}
$$

These are called modified Prüfer variables. The $2 \pi$ ambiguity in $\theta$ is fixed by choosing $\theta(0) \in[0,2 \pi)$ and demanding $\theta(x)$ be continuous in $x$.

Then a straightforward calculation shows (2.1) is equivalent to the pair of equations

$$
\begin{aligned}
\frac{d \theta}{d x} & =k-\frac{V(x)}{k} \sin ^{2}(\theta(x)) \\
\frac{d(\log R)(x)}{d x} & =\frac{1}{2 k} V(x) \sin (2 \theta(x)) .
\end{aligned}
$$

This change of variables is so very useful because if $V=0$, then $\theta(x)=\theta_{0}+k x$, $R(x)=R_{0}$. We will be able to study $V$ as a perturbation about this solution.

As explained in the introduction, one needs to study the asymptotic behavior of the norm of the transfer matrix $T(x, 0)$. For any $\theta_{0}$ in $[0, \pi)$, let $\theta\left(x, \theta_{0}\right)$ solve $(2.3)$ with initial condition $\theta(0)=\theta_{0}$. Then let $R\left(x, \theta_{0}\right)$ solve (2.4) with $R\left(0, \theta_{0}\right)=1$. Then 
Theorem 2.1. For any $\alpha, \beta \in(0, \infty)$ and $\theta_{1} \neq \theta_{2}$, there exists non-zero, finite constants $C_{1}$ and $C_{2}$ (independent of $x$ and $V$ ) so that

$$
C_{1} \max \left(R\left(x, \theta_{1}\right), R\left(x, \theta_{2}\right)\right) \leq\|T(x, 0)\| \leq C_{2} \max \left(R\left(x, \theta_{1}\right), R\left(x, \theta_{2}\right)\right)
$$

for all $k \in(\alpha, \beta)$.

Proof. Define $\|(a, b)\|_{k}^{2}=(k a)^{2}+b^{2}$. Then $\min (1, k)\|(a, b)\| \leq\|(a, b)\|_{k} \leq \max (1, k)$ $\|(a, b)\|$. So defining operator norms in terms of $\|\cdot\|_{k}$, we see $\min \left(k, k^{-1}\right)\|T(x, 0)\|_{k} \leq$ $\|T(x, 0)\| \leq \max \left(k, k^{-1}\right)\|T(x, 0)\|_{k}$, so it suffices to prove (2.5) with $\|\cdot\|_{k}$ rather than $\|\cdot\|$. But

$$
\|T(x, 0)\|_{k} \geq \max \left(R\left(x, \theta_{1}\right), R\left(x, \theta_{2}\right)\right)
$$

is trivial and

$$
\|T(x, 0)\|_{k} \leq\left\{\min \left[\sin \left(\frac{1}{2}\left|\theta_{1}-\theta_{2}\right|\right), \cos \left(\frac{1}{2}\left|\theta_{1}-\theta_{2}\right|\right)\right]\right\}^{-1} \max \left(R\left(x, \theta_{1}\right), R\left(x, \theta_{2}\right)\right)
$$

by the lemma below.

If $\left|\theta_{1}-\theta_{2}\right| \leq \frac{\pi}{2}$ (which can be done by replacing $\theta_{1}$ by $\pi+\theta$, if need be), then this proof shows we can take

$$
\begin{aligned}
& C_{1}=\min \left(\alpha, \beta^{-1}\right), \\
& C_{2}=\max \left(\beta, \alpha^{-1}\right)\left[\sin \left(\frac{1}{2}\left|\theta_{1}-\theta_{2}\right|\right)\right]^{-1} .
\end{aligned}
$$

Lemma 2.2. Let $A$ be a unimodular matrix. Let $u_{\theta}=(\cos (\theta), \sin (\theta))$. Then if $\left|\theta_{1}-\theta_{2}\right| \leq$ $\frac{\pi}{2}$,

$$
\|A\| \leq \sin \left(\frac{1}{2}\left|\theta_{1}-\theta_{2}\right|\right)^{-1} \max \left(\left\|A u_{\theta_{1}}\right\|,\left\|A u_{\theta_{2}}\right\|\right) .
$$

Proof. There exists $\theta_{0}$ so that

$$
\left\|A u_{\theta}\right\|^{2} \geq\|A\|^{2} \sin ^{2}\left(\theta-\theta_{0}\right) .
$$

If $\left|\theta_{1}-\theta_{2}\right|<\frac{\pi}{2}$, for any $\theta_{0}$ at least one of $\left|\sin \left(\theta_{0}-\theta_{i}\right)\right|$ is larger than or equal to $\mid \sin \left(\frac{1}{2}\left(\theta_{1}-\theta_{2}\right) \mid\right.$.

Remark. One might worry that the lemma involves $\|\cdot\|$ and not $\|\cdot\|_{k}$ but $\|A\|_{k}=$ $\left\|\left(\begin{array}{ll}k & 0 \\ 0 & 1\end{array}\right) A\left(\begin{array}{ll}k & 0 \\ 0 & 1\end{array}\right)^{-1}\right\|$ and this product is also unimodular.

For the discrete case, we are interested in solutions of $(0 \leq k \leq \pi)$

$$
u(n+1)+u(n-1)+V(n) u(n)=2 \cos (k) u(n) .
$$

EFGP variables $R(n), \theta(n)$ are defined by

$$
\begin{aligned}
R(n) \cos (\theta(n)) & =u(n)-\cos (k) u(n-1), \\
R(n) \sin (\theta(n)) & =\sin (k) u(n-1) .
\end{aligned}
$$

A priori $\theta(n)$ is only determined $\bmod (2 \pi)$. We will fix this ambiguity later. Noticing that

$$
\begin{gathered}
R(n) \sin (k+\theta(n))=\sin (k) u(n), \\
\frac{u(n)}{u(n-1)}=\frac{\sin (k+\theta(n))}{\sin (\theta(n))} .
\end{gathered}
$$


Similarly,

$$
R(n) \cos (k+\theta(n))=\cos (k) u(n)-u(n-1) .
$$

Thus,

$$
\cot (k+\theta(n))=\frac{\cos (k) u(n)-u(n-1)}{\sin (k) u(n)}
$$

where by definition,

$$
-\cot (\theta(n+1))=\frac{\cos (k) u(n)-u(n+1)}{\sin (k) u(n)} .
$$

Thus, (2.6) is equivalent to

$$
\cot (\theta(n+1))=\cot (k+\theta(n))-\frac{V(n)}{\sin (k)} .
$$

Writing $\bar{\theta}(n) \equiv \theta(n)+k$, we see, using first (2.7) and then (2.8)/(2.9):

$$
\begin{aligned}
R(n+1)^{2} & =\sin ^{2}(k) u(n)^{2}+(u(n+1)-\cos (k) u(n))^{2} \\
& =\sin ^{2}(k) u(n)^{2}+(u(n-1)-\cos (k) u(n)+V(n) u(n))^{2} \\
& =R(n)^{2} \sin ^{2}(\bar{\theta}(n))+R(n)^{2}\left(\cos (\bar{\theta}(n))-\frac{V(n)}{\sin (k)} \sin (\bar{\theta}(n))\right)^{2} \\
& =R(n)^{2}\left[1-\frac{V(n)}{\sin (k)} \sin (2 \bar{\theta}(n))+\frac{V(n)^{2}}{\sin ^{2}(k)} \sin ^{2}(\bar{\theta}(n))\right]
\end{aligned}
$$

We can summarize with the EFGP equations:

$$
\begin{array}{r}
\nu_{k}(n) \equiv-\frac{V(n)}{\sin (k)} ; \quad \bar{\theta}(n)=\theta(n)+k, \\
\cot (\theta(n+1))=\cot (\bar{\theta}(n))+\nu_{k}(n), \\
\frac{R(n+1)^{2}}{R(n)^{2}}=1+\nu_{k}(n) \sin (2 \bar{\theta}(n))+\nu_{k}(n)^{2} \sin ^{2}(\bar{\theta}) .
\end{array}
$$

We will fix the ambiguity in $\theta$ by demanding $\theta(n+1)-\bar{\theta}(n) \in[-\pi, \pi)$. Equation (2.12) can be regarded as analogs of modified Prüfer equations in that if $V=0, R(n)=$ constant, and $\theta(n)=\theta(0)+k n$.

As noted in the introduction, Eggarter arrived at the first version of the EFGP transform by looking at continuum models with $\delta$-function potential $((2.12 \mathrm{~b})$ is especially transparent in this mode). But one could have arrived at it by noting that when $V(n) \equiv 0$, the transfer matrix is powers of $\left(\begin{array}{cc}2 \cos (k) & -1 \\ 1 & 0\end{array}\right)$. This matrix has eigenvalues $e^{ \pm i k}$ and so it must be similar to $\left(\begin{array}{cc}\cos (k) & \sin (k) \\ -\sin (k) & \cos (k)\end{array}\right)$. That similarity transformation will make the powers simple. Indeed,

$$
\left(\begin{array}{rr}
0 & \sin (k) \\
1 & -\cos (k)
\end{array}\right)\left(\begin{array}{cc}
2 \cos (k) & -1 \\
1 & 0
\end{array}\right)=\left(\begin{array}{rr}
\cos (k) & \sin (k) \\
-\sin (k) & \cos (k)
\end{array}\right)\left(\begin{array}{rr}
0 & \sin (k) \\
1 & -\cos (k)
\end{array}\right)
$$

so the transform (2.7) precisely realizes the similarity.

There is an analog of Theorem 2.1. Define $R(n, \theta)$ by requiring $R(1)=1, \theta(1)=\theta$ in $[0, \pi)$. Then 
Theorem 2.3. For any $\alpha \in\left(0, \frac{\pi}{2}\right)$ and $\theta_{1} \neq \theta_{2}$, there exists non-zero, finite constants $C_{1}$ and $C_{2}$ (independent of $x$ and $V$ ) so that for all $k \in(\alpha, \pi-\alpha)$,

$C_{1} \max \left(R\left(n, \theta_{1}\right), R\left(n, \theta_{2}\right)\right) \leq\|T(n-1,0)\| \leq C_{2} \max \left(R\left(n, \theta_{1}\right), R\left(n, \theta_{2}\right)\right)$.

Because of the arccot, (2.12b) is somewhat awkward to deal with. Pastur-Figotin [26] have noted an equivalent form of (2.12b) which is straightforward from

$$
e^{2 i \varphi}=1+\frac{1}{2} \frac{1}{1+i \cot (\varphi)}
$$

viz.,

$$
e^{2 i \theta(n+1)}=e^{2 i \bar{\theta}(n)}+\frac{i \nu_{k}(n)}{2}\left(\frac{\left(e^{2 i \bar{\theta}(n)}-1\right)^{2}}{1-\frac{i \nu_{k}(n)}{2}\left(e^{2 i \bar{\theta}(n)}-1\right)}\right) .
$$

As an application of (2.14) we have

Proposition 2.4. If $\left|\nu_{k}(n)\right|<\frac{1}{2}$, then

$$
|\theta(n+1)-\bar{\theta}(n)| \leq \pi\left|\nu_{k}(n)\right| .
$$

Proof. If $\left|\nu_{k}(n)\right|<\frac{1}{2}$, then (2.14) implies that

$$
\left|e^{2 i \theta(n+1)}-e^{2 i \bar{\theta}(n)}\right| \leq \frac{\left|\nu_{k}(n)\right|}{2} \frac{4}{\frac{1}{2}}=4\left|\nu_{k}(n)\right| .
$$

Since $\left|e^{i \eta}-1\right| \geq \frac{2|\eta|}{\pi}$, we get

$$
|\theta(n+1)-\bar{\theta}(n)| \leq \frac{\pi}{4}\left|e^{2 i \theta(n+1)}-e^{2 i \bar{\theta}(n)}\right|,
$$

and so the claimed result.

Note. Kiselev, Remling, and Simon [20] present a way of defining $R, \theta$ that makes the analogy to the continuum case transparent, makes (2.14) transparent, improves (2.15), and extends to more general $h_{0}$.

\section{Conditional Integrals and A.C. Spectrum}

It follows from $[11,16,17]$ that for both continuum and discrete Schrödinger operators on $[0, \infty)$, we have (see also [33] for a quick proof):

Proposition 3.1. If $S$ is a set of reals so that for each $\lambda \in S, \sup _{x}\left\|T_{\lambda}(x, 0)\right\|<\infty$, then $H$ has purely a.c. spectrum on $S$ in the sense that

(i) For any boundary condition $\theta$ and any $T \subset S$ with $|T|>0$, we have $\rho_{\theta}^{\mathrm{ac}}(T)>0$.

(ii) For any boundary condition $\theta, \rho_{\theta}^{\text {sing }}(S)=0$.

Thus, bounded transfer matrices have important spectral consequences. By Theorems 2.1 and 2.3, if we can show $R(\cdot, \theta)$ remains bounded for two initial $\theta$ 's, we have boundedness of $T$. From this and (2.4), (2.12c), one easily obtains the well-known result that if $\int|V(x)| d x<\infty$, (resp. $\sum|V(n)|<\infty$ ), then the spectrum is purely a.c. in $(0, \infty)$ (resp. $(-2,2))$. Here is a result allowing more general decay, first in the continuum case. 
Theorem 3.2. Fix $k \neq 0$. Suppose that $\lim _{\beta \rightarrow \infty} \int_{x}^{\beta} V(y) e^{2 i k y} d y$ exists and that

$$
W_{k}(x)=\int_{x}^{\infty} V(y) e^{2 i k y} d y
$$

obeys

$$
\int\left|V(x) W_{k}(x)\right| d x<\infty
$$

Then

$$
\varlimsup_{x \rightarrow \infty}\|T(x, 0)\|<\infty
$$

Remarks. 1. This result is not new; it is essentially due to Harris-Lutz [15]. This is a new proof.

2. This result implies that if $V(x)=\sum_{m=1}^{N} a_{m} \sin \left(k_{m} x\right) / x^{\beta}, \beta>\frac{1}{2}$, and $k \neq \pm \frac{1}{2} k_{m}$ for any $m$, then (3.3) holds, and so by Proposition 3.1, the spectrum is purely a.c. except for possible positive eigenvalues of $\left\{\frac{1}{4} k_{m}^{2}\right\}$.

3. In [19], Kiselev proved that if $V(x)=O\left(x^{-\frac{3}{4}-\epsilon}\right)$, then (3.2) holds off a set of Lebesgue measure zero.

Proof. We will show for any $\theta_{0}, R\left(x, \theta_{0}\right)$ is bounded, and then one can appeal to Theorem 2.1 to complete the proof of (3.3). Write $\theta(x)=k x+\varphi(x)$, so by (2.3), $\varphi$ obeys

$$
\frac{d \varphi}{d x}=-\frac{V(x)}{k} \sin ^{2}(k x+\varphi)
$$

By (2.4) (and $R(0)=1$ ),

$$
\begin{aligned}
\log R(x) & =\int_{0}^{x} \frac{1}{2 k} \operatorname{Im}\left[\left(\frac{d W_{k}}{d x}\right) e^{2 i \varphi}\right] d x \\
& =\operatorname{Im}\left[\frac{1}{2 k}\left[W_{k}(x) e^{2 i \varphi(x)}-W_{k}(0) e^{2 i \theta_{0}}\right]-\frac{2 i}{2 k} \int_{0}^{x} W_{k} \frac{d \varphi}{d x} e^{2 i \varphi}\right] d x
\end{aligned}
$$

if we integrate by parts. By hypothesis, $W_{k}(x)$ is bdd so using (3.4),

$$
|\log R(x)| \leq \operatorname{bdd}+\frac{1}{k} \int_{0}^{x}\left|W_{k}(y) V(y)\right| d y
$$

is bounded by (3.2).

Remark. A similar argument proves that

$$
\lim _{x \rightarrow \infty} \theta-k x-\frac{1}{2 k} \int_{0}^{x} V(y) d y
$$

exists. This in turn lets one prove there are complex solutions $\eta_{ \pm}(k, x)$ with

$$
\begin{aligned}
\eta_{ \pm}(k, x) \exp \left(\left(\mp i\left(k x-\frac{1}{2 k} \int_{0}^{x} V(y) d y\right)\right)\right. & \rightarrow 1, \\
\eta_{ \pm}^{\prime}(k, x) \exp \left(\mp i\left(k x-\frac{1}{2 k} \int_{0}^{x} V(y) d y\right)\right) & \rightarrow i k .
\end{aligned}
$$


Notice that if $V \in L^{2}$,

$$
k x-\frac{1}{2 k} \int_{0}^{x} V(y) d y=\int_{0}^{x} \sqrt{k^{2}-V(y)} d y+Q(x),
$$

where $\lim _{x \rightarrow \infty} Q(x)$ exists. So if $V \in L^{2}$, this says that WKB-type solutions exist. This is also what the Harris-Lutz method gives [19].

We are heading toward a proof of

Theorem 3.3. Fix $k \neq 0, \pi$. Suppose $V(n)$ is a discrete potential with

$$
\lim _{B \rightarrow \infty} \sum_{m=n}^{B} V(m) e^{2 i k m}=W_{k}(n)
$$

exists and that

$$
\sum_{n=1}^{\infty}\left|V(n) W_{k}(n)\right|+\left|V(n) W_{k}(n+1)\right|<\infty .
$$

Then

$$
\varlimsup_{n \rightarrow \infty}\|T(n, 0)\|<\infty .
$$

Given a function $f$ on $\{1,2, \ldots\}$, define $(\delta f)(n)=f(n+1)-f(n)$ and note that summation by parts takes the form

$$
\sum_{m=a}^{b} g(m)(\delta f)(m)=-\sum_{m=a}^{b} f(m+1)(\delta g)(m)+(f g)(b+1)-(f g)(a) .
$$

Lemma 3.4. If (3.5) holds for some $k$, then $\sum_{n=1}^{\infty}|V(n)|^{2}<\infty$.

Proof. Since $W$ exists, $V \rightarrow 0$ at $\infty$ and so $V$ is bounded. Thus, writing $V(n)=$ $-e^{-2 i k n}\left(\delta W_{k}\right)(n)$, and summing by parts,

$$
\sum_{n=1}^{B} V(n)^{2}=\mathrm{bdd}+\sum_{n=2}^{B+1} V(n) W_{k}(n) e^{-2 i k n}-\sum_{n=1}^{B} V(n) W_{k}(n+1) e^{-2 i k n}
$$

is bounded by (3.5).

Lemma 3.5. Suppose that $\left\{a_{n}\right\}_{n=1}^{\infty}$ is a real sequence so that

$$
a_{n} \rightarrow 0 \quad \text { as } n \rightarrow \infty
$$

and

$$
\sum_{n=1}^{N} a_{n} \quad \text { is bounded. }
$$

Then $\prod_{n=1}^{N}\left(1+a_{n}\right)$ is bounded.

Proof. By (3.6), $\left|a_{n}\right| \rightarrow 0$, so without loss we can suppose that $\left|a_{n}\right|<1$. Then $\left|1+a_{n}\right| \leq$ $1+a_{n} \leq e^{a_{n}}$ and (3.7) implies the result. 
Proof of Theorem 3.3. By (2.12c), Lemma 3.4, and Lemma 3.5, it suffices to prove that

$$
\sum_{n=1}^{N} \nu_{k}(n) e^{2 i \bar{\theta}(n)} \equiv G(N)
$$

is bounded. Define

$$
\varphi(n)=\theta(n)-k(n-1)=\bar{\theta}(n)-k n .
$$

Proposition 2.4 and Lemma 3.4 imply that for $n$ large

$$
|(\delta \varphi)(n)| \leq \pi\left|\nu_{k}(n)\right|
$$

By the definition (3.8),

$$
\begin{aligned}
G(N) & =-\sum_{n=1}^{N} \delta W_{k}(n)(\sin k)^{-1} e^{2 i \varphi(n)} \\
& =\operatorname{bdd}+(\sin k)^{-1} \sum_{n=1}^{N} W_{k}(n+1) \delta\left(e^{2 i \varphi}\right)(n)
\end{aligned}
$$

But $\left|\delta\left(e^{2 i \varphi}\right)\right| \leq 2|\delta \varphi|$, so by (3.9)

$$
\begin{aligned}
|G(N)-\operatorname{bdd}| & \leq C_{1} \sum_{n=1}^{N}\left|W_{k}(n+1) \nu_{k}(n)\right| \\
& \leq C_{1}\left[\sum_{n=1}^{N}\left|W_{k}(n) \nu_{k}(n)\right|+\left|\nu_{k}(n)\right|^{2}\right]<\infty .
\end{aligned}
$$

Sometimes it is better to use slightly different Prüfer variables. For example, if $R, \theta$ are defined by

$$
\begin{aligned}
u^{\prime}(x) & =\sqrt{E-V(x)} R(x) \cos (\theta(x)), \\
u(x) & =R(x) \sin (\theta(x)),
\end{aligned}
$$

then

$$
\frac{d \log (R)}{d x}=\frac{1}{2} \frac{\partial V}{\partial x} \cos ^{2}(\theta(x)),
$$

from which we see if $V(x) \rightarrow 0$ at infinity and $\frac{\partial V}{\partial x} \in L^{1}$, then solutions are bounded. (This is essentially the proof of Weidmann's theorem [37] in [33].) If one tries out an integration by parts argument, one needs both $\frac{\partial V}{\partial x} \in L^{1}$ and $V \in L^{2}$. 


\section{Bound States for $O\left(x^{-1}\right)$ Potentials}

If $|V(x)|=o\left(x^{-1}\right)$, Eastham-Kalf [8] show that $-\frac{d^{2}}{d x^{2}}+V(x)$ has no positive eigenvalues; more generally, if $\overline{\lim } x|V(x)|=C<\infty$, they show any eigenvalue $\lambda$ must obey $\lambda \leq C^{2}$.

On the other hand, Naboko [24] and Simon [34] have constructed $V(x)$ decaying arbitrarily slower than $x^{-1}$ with eigenvalues dense in [0, $\left.\infty\right)$. In fact, Simon [34] constructed $V(x)$ with $V(x)=O\left(x^{-1}\right)$ so that there are infinitely many eigenvalues with $\lambda_{i} \rightarrow 0$ as long as $\sum \sqrt{\lambda_{i}}<\infty$. In this section, we will handle the borderline case and improve Eastham-Kalf [8] by showing:

Theorem 4.1. Let $V(x)$ obey $C=\varlimsup_{x \rightarrow \infty} x|V(x)|<\infty$. Then there are at most countably many positive eigenvalues $\lambda_{n}$ for which there are solutions $u_{n}$ of

$$
-u_{n}^{\prime \prime}+V(x) u_{n}=\lambda_{n} u_{n}
$$

and $u_{n} \in L^{2}$. Moreover,

$$
\sum_{n} \lambda_{n} \leq \frac{C^{2}}{2} .
$$

Remarks. 1. We do not specify boundary conditions on $V$, that is, (4.1) is a bound on all possible boundary conditions at once.

2. There are $\lambda_{n}$ so that (4.1) holds, but $\sum \sqrt{\lambda_{n}}=\infty$ (e.g., $\lambda_{n}=\frac{3 C^{2}}{\pi^{2} n^{2}}$ ) so there is a gap between Simon's examples and what our bounds allow. We believe the optimal result would be to prove that $\sum_{n} \sqrt{\lambda_{n}} \leq C$.

Without loss of generality by slightly increasing $C$ and looking at $[x, \infty)$, we can suppose that

$$
|V(x)| \leq C(1+|x|)^{-1}
$$

which we henceforth do.

The following is standard (see, e.g., Eastham-Kalf [8]):

Lemma 4.2. If $V$ is bounded and $u$ solves $-u^{\prime \prime}+V u=\lambda u$ and $u \in L^{2}$, then $u^{\prime} \in L^{2}$. In particular, $R\left(x, \theta_{0}\right) \in L^{2}$ for that $\theta_{0}$ with $\left(u(0), u^{\prime}(0)\right)=\left(R_{0} \sin \left(\theta_{0}\right), k R_{0} \cos \left(\theta_{0}\right)\right)$.

Proof.

$$
\begin{aligned}
\int_{0}^{N}\left|u^{\prime}\right|^{2} d x & =\left.u^{\prime} u\right|_{0} ^{N}-\int_{0}^{N} u^{\prime \prime} u d x \\
& =\left.u^{\prime} u\right|_{0} ^{N}+\int_{0}^{N}(\lambda-V) u^{2} d x
\end{aligned}
$$

so if $\lim _{N \rightarrow \infty} \int_{0}^{N}\left|u^{\prime}\right|^{2} d x=\infty$, then $\lim _{N \rightarrow \infty} u^{\prime} u=\infty$, but that implies $u^{2}(N)=$ $u(0)^{2}+2 \int_{0}^{N} u^{\prime} u d x \rightarrow \infty$, contradicting the fact that $u \in L^{2}$.

Lemma 4.3. Let $f$ and $g$ be $C^{1}$ functions on $[1, \infty)$ so that

$$
\left|g^{\prime} f\right|+\left|f^{\prime}\right| \in L^{1}
$$

Then $\int_{0}^{N} f(x) e^{i(k x+g(x))} d x$ is bounded as $N \rightarrow \infty$ for any $k \neq 0$. 
Proof. Write $e^{i k x}=\frac{1}{i k} \frac{d}{d x} e^{i k x}$ and integrate by parts to see that

$$
\left|\int_{1}^{N} f(x) e^{i(k x+g(x))} d x\right| \leq \frac{|f(N)|}{|k|}+\frac{|f(1)|}{|k|}+\frac{1}{|k|} \int_{1}^{N}\left(\left|f^{\prime}\right|+\left|f g^{\prime}\right|\right) d x .
$$

Noting that $|f(N)|=|f(1)|+\int_{1}^{N}\left|f^{\prime}(y)\right| d y$, we see that the integral is bounded.

Remark. If $f(x) \rightarrow 0$ at infinity, this argument shows that $\lim _{N \rightarrow \infty} \int_{1}^{N} f(x) e^{i(k x+g(x))} d x$ exists.

Lemma 4.4. Let $\left\{e_{i}\right\}_{i=1}^{N}$ be a set of unit vectors in a Hilbert space $\mathcal{H}$ so that

$$
\alpha \equiv N \sup _{i \neq k}\left\langle e_{i}, e_{j}\right\rangle<1
$$

Then

$$
\sum_{i=1}^{N}\left|\left\langle g, e_{i}\right\rangle\right|^{2} \leq(1+\alpha)\|g\|^{2}
$$

for any $g \in \mathcal{H}$.

Proof. Let $A$ be the $n \times n$ matrix with $a_{i j}=\left\langle e_{i}, e_{j}\right\rangle$. Note that the Hilbert-Schmidt norm of $A-1$ is bounded by $\left(\sum_{i \neq j}\left\langle e_{i}, e_{j}\right\rangle^{2}\right)^{1 / 2} \leq \alpha$ so (4.3) says that $A$ is invertible. If $B$ is its inverse, then

$$
f_{i}=\sum B_{i j} e_{j}
$$

obeys $\left\langle f_{i}, e_{j}\right\rangle=\delta_{i j}$, and thus

$$
\sum\left\langle g, e_{i}\right\rangle f_{j} \equiv \text { Proj of } g \text { to the span of the } e \text { 's, }
$$

and so

$$
\|g\|^{2} \geq\left\|\sum\left\langle g, e_{i}\right\rangle f_{i}\right\|^{2}
$$

By (4.5), $\left\langle f_{i}, f_{j}\right\rangle=B_{i j}$ and since $\left\langle h, A^{-1} h\right\rangle_{\mathbb{C}^{n}} \geq\|A\|^{-1}\langle h, h\rangle_{\mathbb{C}^{n}}$, we see that

$$
\begin{aligned}
\sum_{i=1}^{n}\left|\left\langle g, e_{i}\right\rangle\right|^{2} & \leq\|A\| \sum_{i, j} \overline{\left\langle g, e_{i}\right\rangle}\left\langle f_{i}, f_{j}\right\rangle\left\langle g, e_{j}\right\rangle \\
& \leq\|A\|\|g\|^{2},
\end{aligned}
$$

which is (4.4).

Proof of Theorem 4.1. It obviously suffices to show for each fixed $N<\infty$ that

$$
\sum_{n=1}^{N} \lambda_{n} \leq \frac{C^{2}}{2}
$$

Define $R_{n}(x)$ to be the $R$ corresponding to the $L^{2}$ solution $u\left(x, \lambda_{n}\right)$. Normalize $u$ so $R_{n}(0)=1$. By Lemma 4.2 , 


$$
\sum_{n=1}^{N}\left|R_{n}(x)\right|^{2} \in L^{1}
$$

SO

$$
\underline{\lim } x \sum_{n=1}^{N}\left|R_{n}(x)\right|^{2}=0
$$

(for if not, eventually $\sum_{n=1}^{N}\left|R_{n}(x)\right|^{2} \geq C x^{-1}$ is not $L^{1}$ ). Thus, we can find $B_{j} \rightarrow \infty$ so that for $n=1, \ldots, N$,

$$
R_{n}\left(B_{j}\right) \leq B_{j}^{-1 / 2}
$$

or

$$
\int_{0}^{B_{j}} \frac{d}{d x}\left(\log R_{n}(y)\right) d y \leq-\frac{1}{2} \ln B_{j}
$$

so by (2.4),

$$
\int_{0}^{B_{j}} V(x) \sin \left(2 \theta_{n}(y)\right) d y \leq-\sqrt{\lambda_{n}} \log B_{j} .
$$

Now consider the Hilbert spaces

$$
\mathcal{H}_{j}=L^{2}\left(\left(0, B_{j}\right),(1+x) d x\right) .
$$

In $\mathcal{H}_{j}$, we have

$$
\|V\|_{\mathcal{H}_{j}}^{2} \leq \int_{0}^{B_{j}} C^{2}(1+|x|)^{-2}(1+x) d x=C^{2} \log \left(B_{j}\right)+O(1) .
$$

Let

$$
e_{n}^{(j)}(y)=\frac{\sin \left(2 \theta_{n}(y)\right)}{(1+|y|)} \frac{1}{\sqrt{N_{n}^{(j)}}} \chi_{\left[0, B_{j}\right]}(y),
$$

where

$$
N_{n}^{(j)}=\int_{0}^{B_{j}} \frac{\sin ^{2}\left(2 \theta_{n}(y)\right)}{(1+|y|)} d y .
$$

Notice that $4 \theta_{n}(y)-4 \sqrt{\lambda_{n}}$ and $2\left(\theta_{n} \pm \theta_{m}\right)-2\left(\sqrt{\lambda_{n}} \pm \sqrt{\lambda_{m}}\right)$ have derivatives that are $O\left(x^{-1}\right)$ by (2.3). Thus by Lemma 4.3,

$$
\int_{0}^{B_{j}} \frac{\sin \left(2 \theta_{n}(y)\right) \sin \left(2 \theta_{m}(y)\right)-\frac{1}{2} \delta_{n m}}{(1+|y|)} d y
$$

are bounded. We conclude that

$$
\begin{array}{r}
N_{i}^{(j)}=\frac{1}{2} \log B_{j}+O(1), \\
\left\langle e_{i}^{(j)}, e_{k}^{(j)}\right\rangle=O\left(\left(\log B_{j}\right)^{-1}\right) \quad i \neq k .
\end{array}
$$

Equations (4.6) and (4.8) imply that

$$
\left\langle V, e_{n}^{(j)}\right\rangle_{\mathcal{H}_{j}} \leq-\sqrt{2 \lambda_{n}}\left(\log B_{j}\right)^{1 / 2}+O(1) .
$$

Since the number $N$ of eigenfunctions is fixed, but $B_{j} \rightarrow \infty$ for $j$ large, Lemma 4.4 applies and 


$$
\sum_{n=1}^{N}\left|\left\langle V, e_{n}^{(j)}\right\rangle_{\mathcal{H}_{j}}\right|^{2} \leq\left(1+O\left(\left(\log B_{j}\right)^{-1}\right)\|V\|_{\mathcal{H}_{j}}^{2} .\right.
$$

But (4.10) and (4.7) then say that

$$
2\left(\sum_{n=1}^{N} \lambda_{n}\right) \log \left(B_{j}\right) \leq C^{2} \log \left(B_{j}\right)+O(1),
$$

SO

$$
\sum_{n=1}^{N} \lambda_{n} \leq \frac{C^{2}}{2}
$$

\section{Sparse Potentials: The Continuum, Absolutely Continuous Case}

Our goal in this section is to prove assertion (1) in Theorem 1.6 and Theorem 1.6' ${ }^{\prime}$ The idea will be to control $\|T(x)\|^{4}$ and then use Theorem 1.3. As explained in Sect. 1, the key is oscillations in $\sin (2 \theta(x))$ for $\theta(x) \sim k x_{n+1}$ for $x$ near $x_{n+1}$. We will realize this using an integration by parts so we need a priori control on objects like $\frac{d\left\|T\left(x_{n}\right)\right\|}{d k}$.

Fix a Pearson potential; $a_{n}$ is assumed to obey $a_{n} \rightarrow 0$ and $x_{n+1}>x_{n}+2 \Delta$. Fix $\theta_{0}$ and solve the modified Prüfer equations for each $k \in(0, \infty)$ to get functions $\theta(x, k)$ and $R(x, k)$ (with initial conditions $\left.\theta(x=0, k)=\theta_{0}, R(x=0, k)=1\right)$. Fix $\Delta$ so $\operatorname{supp}(W) \subset[-\Delta, \Delta]$.

We need two propositions to prepare for bounds in an integration by parts:

Proposition 5.1. Suppose that $\underline{\lim } x_{n+1} / x_{n}>1$. For each $a, b>0$, there exists $a$ constant $C$ so that for each $k \in \overline{(a, b)}$,

$$
\left|\frac{\partial \theta}{\partial k}\left(x_{n}+\Delta\right)\right| \leq C x_{n}
$$

and

$$
\left|\frac{\partial^{2} \theta}{\partial k^{2}}\left(x_{n}+\Delta\right)\right| \leq C x_{n}^{2} .
$$

Moreover, uniformly for $k \in(a, b)$,

$$
\begin{array}{r}
\lim _{x \rightarrow \infty} \frac{1}{x} \frac{\partial \theta}{\partial k}(x)=1, \\
\lim _{x \rightarrow \infty} \frac{1}{x^{2}} \frac{\partial^{2} \theta}{\partial k^{2}}(x)=0 .
\end{array}
$$

Proof. Let

$$
\beta=\inf _{n} \frac{x_{n+1}}{x_{n}}>1
$$

by hypothesis.

As a preliminary, note that if $h, g, f$ are functions on $[a, b], h$ is $C^{1}$ and

$$
h^{\prime}(x)=f(x)+g(x) h(x) .
$$


Then

$$
|h(b)| \leq\left(|h(a)|+(b-a)\|f\|_{\infty}\right) e^{(b-a)\|g\|_{\infty}}
$$

as follows from the exact solution of (5.6):

$$
h(x)=h(x) e^{\int_{a}^{x} g(y) d y}+\int_{a}^{x} f(y) e^{\int_{y}^{x} g(z) d z} d y .
$$

Now let $h(x)=\frac{\partial \theta}{\partial k}(x)$. From (2.3),

$$
\frac{\partial h}{\partial x}=1+\frac{V(x)}{k^{2}} \sin ^{2}(\theta(x))-\frac{V(x)}{k} \sin (2 \theta(x)) h .
$$

This means for $x \in\left(x_{n-1}+\Delta, x_{n}-\Delta\right)$, we have that

$$
\frac{\partial h}{\partial x}=1
$$

By (5.7) and (5.8),

$$
\begin{aligned}
\left|h\left(x_{n}+\Delta\right)\right| & \leq e^{2 C\left|a_{n}\right| \Delta}\left[\left|h\left(x_{n}-\Delta\right)\right|+2 \Delta+2 C\left|a_{n}\right| \Delta\right] \\
& \leq e^{2 C\left|a_{n}\right| \Delta}\left[\left|h\left(x_{n-1}+\Delta\right)\right|+\left(x_{n}-x_{n-1}\right)+2 C\left|a_{n}\right| \Delta\right]
\end{aligned}
$$

where we used (5.9) to go from (5.10) to (5.11). In these equations, $C$ is a constant only depending on $(a, b)$. Throughout this proof, $C$ is such a constant whose value can vary from one equation to the next.

Let $\beta>1$ be given by (5.5). Pick $n_{0}$ so large that for $n \geq n_{0}$ :

$$
\beta^{-1} e^{2\left|a_{n}\right| C \Delta} \leq \frac{1}{2}\left(1+\beta^{-1}\right)
$$

and

$$
\left(1+\frac{2 C\left|a_{n}\right| \Delta}{x_{n}}\right) e^{2 a_{n} C \Delta} \leq 1+\left(\frac{1-\beta^{-1}}{2}\right) .
$$

Since $\beta>1$ and $a_{n} \rightarrow 0$, such an $n_{0}$ exists. Next, pick $D \geq 2$ so

$$
\left|h\left(x_{n_{0}-1}+\Delta\right)\right| \leq D x_{n_{0}-1} .
$$

We claim inductively that for $n \geq n_{0}-1$, we have that

$$
\left|h\left(x_{n}+\Delta\right)\right| \leq D x_{n}
$$

for by (5.14), this holds for $n=n_{0}-1$, and if it holds for $n-1$, then by (5.11) and $x_{n-1} \leq \beta^{-1} x_{n}$,

$$
\begin{aligned}
\left|h\left(x_{n}+\Delta\right)\right| & \leq\left[D x_{n-1}+x_{n}-x_{n-1}+2 C\left|a_{n}\right| \Delta\right] e^{2 C\left|a_{n}\right| \Delta} \\
& \leq x_{n}\left[(D-1) \beta^{-1}+1+\frac{2 C\left|a_{n}\right| \Delta}{x_{n}}\right] e^{2 C\left|a_{n}\right| \Delta} \\
& \leq x_{n}\left[(D-1)\left(\frac{1}{2}\right)\left(1+\beta^{-1}\right)+1+\left(\frac{1-\beta^{-1}}{2}\right)\right] \\
& =x_{n}\left[D-(D-2)\left(\frac{1-\beta^{-1}}{2}\right)\right] \leq D x_{n}
\end{aligned}
$$


since $D \geq 2$. Thus, we've proven (5.15).

Next, let $H(x)=h(x)-x$, so (5.8) implies that

$$
\left|\frac{\partial H}{\partial x}\right| \leq C\left|a_{n}\right|(1+|H|)
$$

on $\left(x_{n}-\Delta, x_{n}+\Delta\right)$. Using (5.7) and (5.15), we conclude (recall the constant $C$ changes from one equation to the next!)

$$
\left|H\left(x_{n}+\Delta\right)-H\left(x_{n}-\Delta\right)\right| \leq C\left|a_{n}\right| x_{n} .
$$

Since $H\left(x_{n-1}+\Delta\right)=H\left(x_{n}-\Delta\right)$, we have that for $n \geq n_{0}$,

$$
\begin{aligned}
\left|\frac{H\left(x_{n}+\Delta\right)}{x_{n}+\Delta}\right| & \leq \frac{C}{x_{n}+\Delta}+\sum_{m=n_{0}}^{n} a_{m} \frac{x_{m}}{\left(x_{n}+\Delta\right)} \\
& \leq \frac{C}{x_{n}+\Delta}+\sum_{m=n_{0}}^{n} a_{m} \beta^{-(n-m)} \rightarrow 0
\end{aligned}
$$

as $n \rightarrow \infty$ since $\beta>1$ and $a_{m} \rightarrow 0$. From this and (5.16), we see that $\left|\frac{H(x)}{x}\right| \rightarrow 0$ as $x \rightarrow \infty$, which proves (5.1). that

To prove (5.2), let $g=\frac{\partial h}{\partial k}=\frac{\partial^{2} \theta}{\partial k^{2}}$. Then differentiating (5.8) with respect to $k$, we see

$$
\begin{aligned}
& \frac{\partial g}{\partial x}=0 \quad \text { on }\left(x_{n-1}+\Delta, x_{n}-\Delta\right) \\
& \frac{\partial g}{\partial x}=A(x)+B(x) h(x)+D(x) g(x)+E(x) h^{2}(x) \quad \text { on }\left(x_{n}-\Delta, x_{n}+\Delta\right)
\end{aligned}
$$

where $A, B, D, E$ are uniformly bounded by $C a_{n}$ on this interval with $C$ uniformly bounded as $k$ runs through $(a, b)$.

Now use (5.7) and (5.1) to see that

$$
\left|g\left(x_{n}+\Delta\right)\right| \leq e^{2 C a_{n} \Delta}\left[g\left(x_{n-1}+\Delta\right)+C a_{n} x_{n}^{2} \Delta\right] .
$$

As above, if $n$ is so large that

$$
\beta^{-2} e^{2 C a_{n} \Delta} \leq \frac{1}{2}\left(1+\beta^{-1}\right) \quad \text { and } \quad\left(C a_{n} \Delta\right) e^{2 C a_{n} \Delta} \leq \frac{1}{2}\left(1-\beta^{-1}\right)
$$

then inductively,

$$
g\left(x_{n}+\Delta\right) \leq C x_{n}^{2}
$$

for $n$ large. This is (5.2). Plugging this into (5.17b), we see that

$$
g\left(x_{n}+\Delta\right) \leq C\left(1+\sum_{m=1}^{n} a_{m} x_{m}^{2}\right)
$$

which yields $\lim _{n \rightarrow \infty} g\left(x_{n}+\Delta\right) / x_{n}^{2}=0$ from which (5.4) is immediate. 
Proposition 5.2. For any $a, b>0$, there is a $C$ so that for all $k \in(a, b)$,

$$
\begin{array}{r}
\log R\left(x_{n}+\Delta\right) \leq C \sum_{m=1}^{n}\left|a_{m}\right|, \\
\frac{\partial \log R}{\partial k}\left(x_{n}+\Delta\right) \leq C \sum_{m=1}^{n}\left|a_{m} x_{m}\right| .
\end{array}
$$

Proof. By (2.4), $\log R(x)$ is constant for $x \in\left(x_{n-1}+\Delta, x_{n}-\Delta\right)$ and

$$
\left|\log R\left(x_{n}+\Delta\right)-\log R\left(x_{n}-\Delta\right)\right| \leq 2 k^{-1}\left|a_{n}\right| \int W(y) d y,
$$

so (5.18) holds with $C=2 \min (k)^{-1} \int W(y) d y$.

From (2.4), we have

$$
\frac{\partial}{\partial x} \frac{\partial}{\partial k}(k \log R)=V(x) \cos (2 \theta(x)) \frac{\partial \theta}{\partial k},
$$

so that the bound (5.1) implies (5.9).

As a final preliminary, we note that

Lemma 5.3. Suppose that $\underline{\lim } x_{n+1} / x_{n}>1$. Then for a constant $C$,

$$
\sum_{n=1}^{\infty} \sum_{m \leq n}\left|a_{n} a_{m}\right| \frac{x_{m}}{x_{n}} \leq C \sum_{n=1}^{\infty} a_{n}^{2} .
$$

Proof. Let $\beta=\lim x_{n+1} / x_{n}$. Pick $1<\gamma<\beta$. Then for $m \leq n, x_{m} / x_{n} \leq C \gamma^{-|m-n|}$. Thus, the lemma follows from Young's inequality that

$$
T(a)_{n} \equiv \sum_{m} \gamma^{-|m-n|} a_{m}
$$

is bounded from $\ell^{2}$ to $\ell^{2}$ for any $\gamma>1$.

Proof of Theorem $1.6^{\prime}$. Let $g$ be a non-negative $C^{\infty}$-function compactly supported on $(0, \infty)$. We will prove that

$$
\sup _{n} \int g(k) R\left(k, x_{n}+\Delta\right)^{4} d k<\infty
$$

Proving this for two values of $\theta_{0}$ and appealing to Theorem 2.1 gets a uniform bound on $\int g(k)\left\|T\left(0, x_{n}+\Delta\right)\right\|^{4} d k$. Theorem 1.3 then proves pure absolute continuity of the spectrum on $(0, \infty)$.

Let $B_{n}=\int g(k) R\left(x_{n}+\Delta\right)^{4} d k$. Notice that by (2.4), $R\left(x_{n-1}+\Delta\right)=R\left(x_{n}-\Delta\right)$ and

$$
R\left(x_{n}+\Delta\right)^{4}=R\left(x_{n}-\Delta\right)^{4} \exp \left(Q_{n}\right),
$$

where

$$
Q_{n}=\frac{2}{k} \int_{-\Delta}^{\Delta} a_{n} W(y) \sin \left(2 \theta\left(x_{n}+y\right)\right) d y .
$$

Since $k^{-1}$ and $a_{n}$ are bounded, $Q_{n}$ is uniformly bounded in $n$, and so 


$$
\begin{aligned}
\exp \left(Q_{n}\right) & \leq 1+Q_{n}+C Q_{n}^{2} \\
& \leq 1+Q_{n}+C a_{n}^{2}
\end{aligned}
$$

(where again $C$ is a constant that varies from formula to formula).

For $y \in(-\Delta, \Delta)$, we have by $(2.3)$

$$
\left|\theta\left(x_{n}+y\right)-\tilde{\theta}_{n}(y)\right| \leq C a_{n},
$$

where

$$
\tilde{\theta}_{n}(y)=\theta\left(x_{n-1}+\Delta\right)+k\left(x_{n}+y-x_{n-1}-\Delta\right)
$$

so

$$
\left|Q_{n}-\frac{2}{k} \int_{-\Delta}^{\Delta} a_{n} W(y) \sin \left(2 \tilde{\theta}_{n}(y)\right) d y\right| \leq C a_{n}^{2} .
$$

By (5.21)-(5.23),

$$
B_{n} \leq B_{n-1}\left(1+C a_{n}^{2}\right)+E_{n},
$$

where

$$
E_{n}=a_{n} \int_{-\Delta}^{\Delta} d y \int \frac{2 g(k)}{k} R\left(x_{n-1}+\Delta, k\right)^{4} W(y) \sin \left(\tilde{\theta}_{n}(y)\right) d k
$$

Notice that we're implementing our basic strategy: We separate out the second-order terms (which will present no problem since $\prod_{n=1}^{\infty}\left(1+C a_{n}^{2}\right)<\infty$ ) and need to control the first-order terms where we have an explicit highly oscillatory factor since $\theta_{n} \sim k x_{n}$.

Now

$$
\frac{\partial \tilde{\theta}_{n}}{\partial k}(y)=x_{n}+y-x_{n-1}-\Delta+\frac{\partial \theta\left(x_{n-1}+\Delta\right)}{\partial k}>\frac{1}{2} x_{n}
$$

for $n$ large by the bound (5.3).

Thus, we can write

$$
\sin \left(\tilde{\theta}_{n}(y)\right)=\frac{1}{\frac{\partial \tilde{\theta}_{n}}{\partial k}} \frac{\partial}{\partial k}\left(-\cos \left(\tilde{\theta}_{n}(y)\right)\right)
$$

and integrate by parts.

After integration by parts, we have three terms

$$
\begin{aligned}
& E_{n}^{(1)} \text { coming from } \frac{\partial\left[k^{-1} g(k)\right]}{\partial k}, \\
& E_{n}^{(2)} \text { coming from } \frac{\partial R^{4}}{\partial k}, \\
& E_{n}^{(3)} \text { coming from } \frac{\partial}{\partial k}\left(\frac{1}{\frac{\partial \tilde{\theta}}{\partial k}}\right) .
\end{aligned}
$$

For the $E_{n}^{(1)}$ term, we can bound $R^{4}$ as follows using (5.18) and

$$
x_{n} \geq C \beta^{n} .
$$

By (5.10), for $n$ large, 


$$
\begin{aligned}
R^{4} & \leq C \exp \left(C \sum_{m=1}^{n} a_{m}\right) \\
& \leq C \exp \left(\frac{n}{2} \ln (\beta)\right),
\end{aligned}
$$

since $a_{n} \rightarrow 0$. Thus, by (5.19) and (5.26),

$$
E_{n}^{(1)} \leq C \beta^{n / 2} \beta^{-n}=C \beta^{-n / 2} .
$$

For the $E_{n}^{(2)}$ term, we use $\frac{\partial R^{4}}{\partial k}=R^{4} \frac{\partial \log R}{\partial k},(5.19)$, and (5.25) to see that

$$
E_{n}^{(2)} \leq C B_{n-1} b_{n}
$$

where

$$
b_{n}=\sum_{m=1}^{n-1} a_{n} a_{m} \frac{x_{m}}{x_{n}} .
$$

Note now that by $\sum a_{n}^{2}<\infty$ and Lemma 5.3, we have

$$
\sum_{n=1}^{\infty} b_{n}<\infty
$$

For the $E_{n}^{(3)}$ term, we use (5.25) and (5.17c) to see that

$$
E_{n}^{(3)} \leq B_{n-1} c_{n},
$$

where

$$
c_{n}=C a_{n} \frac{\left(1+\sum_{m=1}^{n-1} a_{m} x_{m}^{2}\right)}{x_{n}^{2}} .
$$

As in the proof of Lemma 5.3,

$$
\sum c_{n}<\infty
$$

By (5.24) and the above estimates on $E_{n}^{(i)}$,

$$
\max \left(B_{n}, 1\right) \leq\left(1+C a_{n}^{2}+C b_{n}+C c_{n}+C \beta^{-n / 2}\right) \max \left(B_{n-1}, 1\right) .
$$

By hypothesis, $\sum a_{n}^{2}<\infty$, and by (5.28-5.29), $\sum b_{n}+c_{n}<\infty$. Thus

$$
\prod_{n=1}^{N}\left(1+C a_{n}^{2}+C b_{n}+C c_{n}+C \beta^{-n / 2}\right)
$$

is bounded and consequently, so is $B_{n}$.

It is easy to see that the methods of this section extend to prove:

Theorem 5.4. Suppose $V(x)=\sum W_{n}\left(x-x_{n}\right)$, where

(i) $\varlimsup x_{n} / x_{n+1}<1$,

(ii) $\operatorname{supp} W_{n} \subset[-\Delta, \Delta]$ for some fixed $\Delta$,

(iii) $\sum_{n} \int\left|W_{n}(y)\right|^{2} d y<\infty$.

Then $-\frac{d^{2}}{d x^{2}}+V(x)$ has purely a.c. spectrum on $(0, \infty)$. 


\section{Sparse Potentials: The Continuum, Singular Continuous Case}

In this section, we will prove assertion (2) in Theorem 1.6. The idea will be to force $\left\|T\left(k^{2}, x_{n}\right)\right\|$ to infinity for almost all $k$ and suitable $x_{n}$. To do this, we will need to isolate a strictly positive second-order term and show that these second-order terms then dominate the first-order terms because of oscillations.

Here is a warm-up problem to show this cancellation mechanism. Let $X_{n}$ be independent, identically distributed random variables taking the values \pm 1 with probability $\frac{1}{2}$. Let $\epsilon>0$ and let $a_{n}$ be a sequence going to zero as $n \rightarrow \infty$. Finally, let

$$
Y_{n}=\sum_{m=1}^{n}\left(\epsilon a_{m}^{2}+a_{m} X_{m}\right)
$$

Suppose that $\sum a_{n}^{2}=\infty$. We claim there exists a subsequence $n(i) \rightarrow \infty$, so with probability 1 ,

$$
\lim _{i \rightarrow \infty} Y_{n(i)}=\infty .
$$

The reason (6.1) holds is that by the central limit theorem $\sum_{m=1}^{n} a_{n} X_{n}$ is typically not more negative than $O\left(-\sqrt{\sum a_{n}^{2}}\right)$ and, because of the square root, this is smaller than $\epsilon \sum_{m=1}^{n} a_{n}^{2}$.

To make a proof, notice that since $\sum_{m=1}^{n} a_{m}^{2} \rightarrow \infty$, we can choose $n(i)$ so that $\sum_{m=1}^{n(i)} a_{m}^{2} \geq i^{2}$. By a Tschbechev inequality,

$$
\operatorname{Prob}\left(\sum_{1}^{n(i)} a_{m} X_{m} \geq \frac{\epsilon}{2} \sum_{1}^{n(i)} a_{m}^{2}\right) \leq \frac{\left\|\sum_{1}^{n(i)} a_{m} X_{m}\right\|^{2}}{\left(\frac{\epsilon}{2} \sum_{1}^{n(i)} a_{m}^{2}\right)^{2}}=\frac{4}{\epsilon^{2}} \frac{1}{\sum_{1}^{n(i)} a_{m}^{2}} \leq \frac{4}{\epsilon^{2} i^{2}} .
$$

$\sum \frac{1}{i^{2}}<\infty$, so by the Borel-Cantelli lemma, with probability 1 , eventually

$$
\sum_{1}^{n(i)} a_{m} X_{m} \leq \frac{\epsilon}{2} \sum_{1}^{n(i)} a_{n}^{2}
$$

and thus eventually,

$$
Y_{n(i)} \geq \frac{\epsilon}{2} \sum_{1}^{n(i)} a_{m}^{2}
$$

diverges.

The usual Kolmogorov stopping argument that lets one prove things without subsequences isn't obviously applicable here in a situation where we assume no regularity on the $a_{m}$ 's (see Sect. 8 for the case $a_{m}=m^{-\alpha}$ ). Since a subsequence suffices for our application, we have not tried to push the argument through to get $\lim Y_{n}=\infty$, even in the toy problem.

Notice that independence of the $X_{n}$ 's was not needed; rather, it suffices to have enough control of $E\left(X_{n} X_{m}\right)$ to show that the first-order term is small compared to the second-order term. In the case at hand, we will use integration by parts in $k$ as we did in the last section to get this control.

We summarize the key to the above argument with

Lemma 6.1. Let $P_{n}, Q_{n}$ be random variables so that 
(i) $P_{n}(x) \geq \alpha_{n}>0$ for a.e. $x$ and positive reals $\alpha_{n}$,

(ii) $\sum \alpha_{n}^{-1} \operatorname{Exp}\left(\left|Q_{n}\right|\right)<\infty$,

(iii) $\lim _{n \rightarrow \infty} \alpha_{n}=\infty$.

Then $P_{n}(x)+Q_{n}(x) \rightarrow \infty$ for a.e. $x$. If (ii) is replaced with

(ii') $\underline{\lim }_{n \rightarrow \infty} \alpha_{n}^{-1} \operatorname{Exp}\left(\left|Q_{n}\right|\right)=0$, then there exists a subsequence $n(i)$ so that $P_{n(i)}(x)+$ $Q_{n(i)}(x) \rightarrow \infty$ for a.e. $x$.

Proof. If (ii') holds, we can find a subsequence so that (ii) holds. Thus, it suffices to prove the result assuming (ii).

By (ii), $\sum \alpha_{n}^{-1}\left|Q_{n}(x)\right|<\infty$ for a.e. $x$. In particular, $\alpha_{n}^{-1} Q_{n}(x) \rightarrow 0$ so $P_{n}+Q_{n} \geq$ $\alpha_{n}\left[1-\alpha_{n}^{-1}\left|Q_{n}(x)\right|\right] \rightarrow \infty$.

We will also need the following lemma:

Lemma 6.2. Suppose that $B_{n}, \alpha_{n}, \beta_{n} \geq 0$ are real numbers and that

$$
B_{n} \leq B_{n-1}+2 \alpha_{n} \sqrt{B_{n-1}}+\beta_{n} \quad(n \geq 1) .
$$

Then,

$$
\sqrt{B_{n}} \leq \sqrt{B_{0}}+\sum_{k=1}^{n} \alpha_{k}+\sqrt{\sum_{k=1}^{n} \beta_{k}} .
$$

Proof. We give a proof by induction. Equation (6.2) holds for $n=0$. Let $a_{n}=\sum_{k=1}^{n} \alpha_{k}$, $b_{n}=\sum_{k=1}^{n} \beta_{k}$. By the induction hypothesis,

$$
\sqrt{B_{n-1}} \leq \sqrt{B_{0}}+a_{n-1}+\sqrt{b_{n-1}} .
$$

Equation (6.2) implies that

$$
B_{n} \leq\left(\sqrt{B_{n-1}}+\alpha_{n}\right)^{2}+\beta_{n}
$$

So by (6.4),

$$
\begin{aligned}
B_{n} & \leq\left(\sqrt{B_{0}}+a_{n}+\sqrt{b_{n-1}}\right)^{2}+\beta_{n} \\
& \leq\left(\sqrt{B_{0}}+a_{n}\right)^{2}+b_{n}+2 \sqrt{b_{n-1}}\left(\sqrt{B_{0}}+a_{n}\right) \\
& \leq\left(\sqrt{B_{0}}+a_{n}+\sqrt{b_{n}}\right)^{2},
\end{aligned}
$$

proving (6.3) inductively.

So fix a Pearson potential with $\sum a_{n}^{2}=\infty$. Fix $\theta_{0}$ and let $R(x, k)$ be the solution of (2.3/2.4). Let

$$
Y_{n}(k)=\log R\left(x_{n}+\Delta, k\right)
$$

and

$$
\delta Y_{n}(k)=Y_{n}(k)-Y_{n-1}(k) .
$$

By (2.4), 


$$
\delta Y_{n}(k)=\frac{a_{n}}{2 k} \int_{-\Delta}^{\Delta} W(y) \sin 2 \theta\left(x_{n}+y\right) d y .
$$

As in Sect. 5, we write

$$
\tilde{\theta}_{n}(y)=\theta\left(x_{n-1}+\Delta\right)+k\left(x_{n}+y-x_{n-1}-\Delta\right) .
$$

But we expand $\theta$ to the next order by letting

$$
\theta_{n}^{(1)}(y)=-\frac{a_{n}}{k} \int_{-\Delta}^{y} W(y) \sin ^{2}\left(\tilde{\theta}_{n}(y)\right) d y .
$$

Then by (2.3),

$$
\theta\left(x_{n}+y\right)=\tilde{\theta}_{n}(y)+\theta_{n}^{(1)}(y)+O\left(a_{n}^{2}\right)
$$

so by (6.5),

$$
\begin{aligned}
\delta Y_{n}(k) & =a_{n} X_{n}^{(1)}+a_{n}^{2} S_{n}+O\left(a_{n}^{3}\right), \\
X_{n}^{(1)} & =\frac{1}{2 k} \int_{-\Delta}^{\Delta} W(y) \sin \left(2 \tilde{\theta}_{n}(y)\right) d y, \\
S_{n} & =\frac{1}{k} \int_{-\Delta}^{\Delta} W(y) \cos \left(2 \tilde{\theta}_{n}(y)\right)\left[\frac{\theta_{n}^{(1)}(y)}{a_{n}}\right] .
\end{aligned}
$$

In the formula for $\theta_{n}^{(1)}$, use

$$
\sin ^{2}\left(\tilde{\theta}_{n}(y)\right)=\frac{1}{2}\left(1-\cos \left(2 \tilde{\theta}_{n}(y)\right)\right) .
$$

The cos term from this formula when plugged into $(6.7 \mathrm{c})$ gives

$$
\begin{aligned}
\frac{1}{2} k^{2} \int_{-\Delta}^{\Delta} W(y) \cos \left(2 \tilde{\theta}_{n}(y)\right) & \left(\int_{-\Delta}^{y} W(s) \cos \left(2 \tilde{\theta}_{n}(s)\right)\right) d y \\
& =\frac{1}{4 k^{2}}\left(\int_{-\Delta}^{\Delta} W(y) \cos \left(2 \tilde{\theta}_{n}(y)\right) d y\right)^{2} .
\end{aligned}
$$

We lump the contribution of the $\frac{1}{2}$ term with the first-order term. Defining $X(y)=$ $\int_{-\Delta}^{y} W(s) d s$, we find

$$
\delta Y_{n}(k)=\left[a_{n}^{2} Z_{n}(k)+a_{n} X_{n}(k)\right]+O\left(a_{n}^{3}\right),
$$

where

$$
\begin{aligned}
& Z_{n}(k)=\frac{1}{4 k^{2}}\left(\int_{-\Delta}^{\Delta} W(y) \cos \left(2 \tilde{\theta}_{n}(y)\right) d y\right)^{2} \\
& X_{n}(k)=\frac{1}{2 k} \int_{-\Delta}^{\Delta}\left[W(y) \sin \left(2 \tilde{\theta}_{n}(y)\right)-\frac{a_{n} W(y) X(y)}{2 k} \cos \left(2 \tilde{\theta}_{n}(y)\right)\right] d y .
\end{aligned}
$$

In (6.9), the $O\left(a_{n}^{3}\right)$ means an error bounded by $C a_{n}^{3}$, where $C$ is a finite constant for $k \in[a, b]$ any compact subinterval of $(0, \infty)$.

Define

$$
\widetilde{W}(k)=\int_{-\Delta}^{\Delta} W(y) e^{2 i k y} d y .
$$


Then,

$$
Z_{n}(k)=\frac{1}{8 k^{2}}|\widetilde{W}(k)|^{2}+\tilde{X}_{n}(k)
$$

where

$$
\tilde{X}_{n}(k)=\frac{1}{8 k^{2}}|\widetilde{W}(k)|^{2} \cos \left(4\left(\tilde{\theta}_{n}(0, k)+\varphi(k)\right),\right.
$$

where $\varphi(k)=\frac{1}{2} \operatorname{Arg}(\widetilde{W}(k))$.

For let $\tilde{\theta}_{n}(y)=\tilde{\theta}_{n}(0)+k y$. If $\widetilde{W}(k)=|\widetilde{W}(k)| e^{2 i \varphi(k)}$, then

$$
\begin{aligned}
Z_{n}(k) & =\frac{1}{4 k^{2}}\left(\operatorname{Re} \int_{-\Delta}^{\Delta} W(y) e^{2 i\left(\tilde{\theta}_{n}(0)+k y\right)} d y\right)^{2} \\
& =\frac{1}{4 k^{2}}|\widetilde{W}(k)|^{2} \cos ^{2}\left(2\left(\tilde{\theta}_{n}(0, k)+\varphi(k)\right)\right) .
\end{aligned}
$$

Proof of Theorem 1.6, Part (2). Let

$$
\begin{aligned}
P_{n}(k) & =\frac{1}{8 k^{2}}|\widetilde{W}(k)|^{2} \sum_{m=1}^{n} a_{m}^{2}, \\
Q_{n}(k) & =Y_{n}(k)-P_{n}(k) ; \quad \delta Q_{n}(k)=Q_{n}(k)-Q_{n-1}(k),
\end{aligned}
$$

so

$$
\delta Q_{n}(k)=a_{n}^{2} \tilde{X}_{n}(k)+a_{n} X_{n}(k)+O\left(a_{n}^{3}\right) .
$$

Let $g$ be a $C^{\infty}$-function compactly supported in $\{k \in(0, \infty) \mid \widetilde{W}(k) \neq 0\}$. Let

$$
\begin{aligned}
& B_{n}=\int g(k)\left|\sum_{m=1}^{n} a_{m} X_{m}(k)\right|^{2} d k, \\
& \tilde{B}_{n}=\int g(k)\left|\sum_{m=1}^{n} a_{m}^{2} \tilde{X}_{m}(k)\right|^{2} d k .
\end{aligned}
$$

We will prove that

$$
\sqrt{B_{n}} / \sum_{m=1}^{n} a_{m}^{2} \rightarrow 0
$$

as $n \rightarrow \infty$, and similarly for $\widetilde{B}_{n}$. Since $\sum_{m=1}^{n} a_{m}^{3} / \sum_{m=1}^{n} a_{m}^{2} \rightarrow 0$ (on account of $a_{n} \rightarrow 0$ and $\left.\sum_{m=1}^{n} a_{m}^{2} \rightarrow \infty\right),(6.12)$ and the Schwartz inequality imply that

$$
\int g(k)\left|Q_{n}(k)\right| d k / \sum_{m=1}^{n} a_{m}^{2} \rightarrow 0,
$$

so by Lemma 6.1 and $\inf _{k \in \operatorname{Supp}} \frac{|\widetilde{W}(k)|}{8 k^{2}}>0$ implies that there is a subsequence $n(i)$ so that $Y_{n(i)}(k) \rightarrow \infty$ for a.e. $k$ in supp $g$. By doing this for two values of $\theta_{0}$ and using Theorem 2.1 and Theorem 1.1, we conclude there is no a.c. spectrum on supp $g$.

Since $\widetilde{W}$ is an entire function, it has isolated zeros and thus, this argument shows $\sigma_{\text {ac }}$ is empty. By Theorem 1.4, $\sigma_{\mathrm{pp}} \cap(0, \infty)$ is empty, and an elementary argument proves that $\sigma(H) \supset[0, \infty)$. So the spectrum on $(0, \infty)$ is purely singular continuous. It thus suffices to prove (6.12) (the proof for $\tilde{B}_{n}$ is essentially identical). 
Let $M_{n-1}(k)=\sum_{m=1}^{n-1} a_{m} X_{m}(k)$. Then

$$
B_{n} \leq B_{n-1}+\left|\int g(k) M_{n-1}(k) a_{n} X_{n}(k)\right|+C a_{n}^{2}
$$

for a suitable constant $C$. Now $X_{n}$ has $\cos \left(2 \tilde{\theta}_{n}(y)\right)$ and $\sin \left(2 \tilde{\theta}_{n}(y)\right)$ terms. As in the last section, we write those as a suitable $\left[d \tilde{\theta}_{n}(y, k) / d k\right]^{-1} \frac{d}{d k}[\ldots]$ and integrate by parts and get three terms:

One coming from $\frac{\partial\left[k^{-1} g(k)\right]}{\partial k} \partial k$. Noting that $\left|M_{n}(k)\right| \leq C n$, we have that this is bounded by $\frac{C n}{x_{n}}$.

One coming from $\frac{\partial M_{n}(k)}{\partial k}$. Using (5.1), this term is bounded by

$$
C \sum_{m=1}^{n-1} a_{n} a_{m} \frac{x_{m}}{x_{n}}
$$

One coming from $L_{n}=\left[\frac{\partial^{2} \theta_{n}}{\partial k^{2}}\right] /\left[\frac{\partial \theta_{n}}{\partial k}\right]^{2}$. As in the last section, this $L_{n}$ is bounded by $C\left(\sum_{m=1}^{n-1} a_{m} x_{m}^{2}\right) / x_{n}^{2}$. We can use the Schwartz inequality to control $\int g(k)\left|M_{n}(k)\right| d k$, and so bound this term by $C \sqrt{B_{n-1}} a_{n} \sum_{m=1}^{n-1} a_{m} x_{m}^{2} / x_{n}^{2}$. The net result is the bound

$$
B_{n} \leq B_{n-1}+2 \alpha_{n} \sqrt{B_{n-1}}+\beta_{n},
$$

where

$$
\alpha_{n}=C \sum_{m=1}^{n-1}\left|a_{n} a_{m}\right| \frac{x_{m}^{2}}{x_{n}^{2}}
$$

and

$$
\beta_{n}=C\left[a_{n}^{2}+\frac{n}{x_{n}}+\sum_{m=1}^{n-1} a_{n} a_{m} \frac{x_{m}}{x_{n}}\right] .
$$
that

By the argument in Lemma 5.3 with $x_{n-1} / x_{n} \rightarrow 0$ and $\sum_{m=1}^{\infty} a_{n}^{2} \rightarrow \infty$, we see

$$
\sum_{m=1}^{n} \alpha_{m} / \sum_{m=1}^{n} a_{m}^{2} \rightarrow 0
$$

and that

$$
\sum_{m=1}^{n} \beta_{m} \leq C\left(1+\sum_{m=1}^{n} a_{m}^{2}\right)
$$

so

$$
\sqrt{\sum_{m=1}^{n} \beta_{m}} / \sum_{m=1}^{n} a_{m}^{2} \rightarrow 0 .
$$

Lemma 6.2 and (6.13-6.15) imply (6.12).

One can modify this construction to make examples of decaying potentials for which the associated Schrödinger operator has regions of a.c. spectrum and regions of s.c. spectrum. The idea is to arrange that $\widetilde{W}(k)$ vanishes in a whole interval so that even though 
$a_{n} \notin \ell_{2}$, we have a.c. spectrum for those $k$. Of course, $\widetilde{W}(k)$ cannot vanish if $W$ has compact support, so we will take the bump functions of increasing support converging toward a function whose Fourier transform vanishes in an interval. So, let $S=[a, b] \subset(0, \infty)$. Let $f$ be an even Schwartz class function that vanishes if $k^{2} \in S$ and is strictly positive on $[0, \infty) \backslash S$.

Let $a_{n}=n^{-1 / 2}, x_{n}=(n !)^{2}, \Delta_{n}=n^{-1 / 12}$. Notice that $\sum a_{n}^{2}=\infty$. Define

$$
\begin{array}{r}
\tilde{f}(x)=\frac{1}{4 \pi} \int \exp (-2 i k x) f(k) d k, \\
W_{n}(x)=\tilde{f}(x) \chi_{\left(-\Delta_{n}, \Delta_{n}\right)}(x)
\end{array}
$$

and

$$
V(x)=\sum_{n} a_{n} W_{n}\left(x-x_{n}\right) .
$$

We are heading toward:

Theorem 6.3. The half-axis Schrödinger operator $-\frac{d^{2}}{d x^{2}}+V(x)$ has purely singular spectrum on $(0, \infty) \backslash S$ and purely a.c. spectrum on $S$.

Lemma 6.4. For any $m>0$, there exists a constant $C_{m}$ with

$$
\left|f(k)-\int e^{2 i k x} W_{n}(x) d x\right| \leq C_{m} n^{-m} .
$$

Proof. Let $f_{n}(k)=\int e^{-2 i k x} W_{n}(x) d x$. Then

$$
f_{n}(k)=\frac{1}{2 \pi} \int \frac{\sin \left(\Delta_{n}\left(k-k^{\prime}\right)\right)}{\left(k-k^{\prime}\right)} f\left(k^{\prime}\right) d k^{\prime},
$$

so the left side of (6.16) is

$$
\frac{1}{2 \pi}\left|\int \frac{\sin \Delta_{n}\left(k-k^{\prime}\right)}{k-k^{\prime}}\left[f(k)-f\left(k^{\prime}\right)\right] d k^{\prime}\right|,
$$

which has the form

$$
\left|\int g(y, k) \sin \Delta_{n} y d y\right|,
$$

where $g(y, k)$ is Schwartz space in $y$ with bounds (including bounds on derivatives) uniform in $k$. If we integrate by parts $12 m$ times, we will get (6.16).

Proposition 5.1 extends with no change. In the region where $f(k) \neq 0$, the analysis earlier in this section shows that $\log R\left(x_{n(i)}+\Delta_{n(i)}\right) \rightarrow \infty$ for a.e. $k$ and a suitable subsequence $x_{n(i)}$, so we know the spectrum in $(0, \infty) \backslash S$ is purely singular continuous.

On the other hand, if $g$ is $C^{\infty}$ supported in $S$, we claim that

$$
\sup _{n} \int g(k) R\left(k, x_{n}+\Delta_{n}\right)^{4} d k<\infty \text {. }
$$

The proof is similar to that in the last section. In place of (5.22), we need to use

$$
\exp \left(Q_{n}\right) \leq 1+Q_{n}+\frac{1}{2} Q_{n}^{2}+O\left(a_{n}^{3}\right) .
$$


As in this section, $Q_{n}^{2}$ has a term $a_{n}^{2}\left|\widetilde{W}_{n}(k)\right|^{2} / 8 k^{2}$ and oscillatory terms that we can integrate by parts. Noting that

$$
\sum_{m=1}^{n-1} a_{n} a_{m} \frac{x_{m}}{x_{n}} \leq n^{-2} n^{-1 / 2} \sum_{m=1}^{n-1} m^{-1 / 2} \leq C n^{-2}
$$

is still summable and that $\sum a_{n}^{2}\left|\widetilde{W}_{n}(k)\right|^{2}$ is summable by Lemma 6.4, we obtain (6.17).

\section{Sparse Potentials: The Discrete Case}

In this section, we will sketch the proof of Theorem 1.7. The proof follows closely that in the last two sections with (2.12) replacing (2.3/2.4). We will make use of (2.14), the Pastur-Figotin form of (2.12b).

Fix $\alpha>0$ and pick $k \in(\alpha, \pi-\alpha)$ and then $N$ so large that for all such $k,\left|\nu_{k}(n)\right|<\frac{1}{2}$ for $n \geq N_{0}$. Equation (2.14) can then be effectively used to prove the analogs of (5.1/5.2), that is,

$$
\left|\frac{\partial \theta}{\partial k}\left(x_{n}\right)\right| \leq C x_{n} \quad\left|\frac{\partial^{2} \theta}{\partial k^{2}}\left(x_{n}\right)\right| \leq C x_{n}^{2} .
$$

Equation (2.12c) can be rewritten

$$
\log R(n+1)-\log R(n)=\frac{1}{2} \log \left(1+\nu_{k}(n) \sin (2 \bar{\theta})+\nu_{k}(n)^{2} \sin ^{2}(\bar{\theta})\right) .
$$

This implies the bound

$$
\log R\left(x_{n}\right) \leq C \sum_{m=1}^{n}\left|a_{m}\right|
$$

Next notice that

$$
1+\alpha \sin (2 \theta)+\alpha^{2} \sin ^{2}(\theta)=\left(1+\frac{1}{2} \alpha \sin (2 \theta)\right)^{2}+\alpha^{2} \sin ^{4}(\theta) .
$$

This provides a uniform bound on the argument of the $\log (\cdot)$ in (7.2), and so allows one to prove

$$
\left|\frac{\partial}{\partial k} \log R\left(x_{n}\right)\right| \leq C \sum_{m=1}^{n} a_{n} x_{m} .
$$

With these tools, the proof of assertion (1) of Theorem 1.6 is similar to that in Sect. 5, only a little simpler since (2.12c) implies

$$
R(n+1)^{4} \leq R(n)^{4}\left(1+\nu_{k}(n) \sin (2 \bar{\theta}(n))+C_{n} a_{n}^{2}\right) .
$$

The same integration by parts used in Sects. 5 and 6 shows that

$$
\int g(k) R(n, k)^{4} \nu_{k}(n) \sin (2 \theta(n)) d k=C\left(b_{n}+c_{n}+B^{-n / 2}\right)\left(1+\int g(k) R(n, k)^{4} d k\right)
$$

with $b_{n}=\sum_{m-1}^{n-1} a_{n} a_{m} x_{m} / x_{n}$ and $c_{n}$ is like $b_{n}$ with $x_{m}^{2} / x_{n}^{2}$ replacing $x^{m} / x^{n}$. As in Sect. 5, this proves assertion (1) in Theorem 1.7.

To prove assertion (2), we must identify a strictly positive second-order term. We write 


$$
\begin{aligned}
& \log \left(1+\alpha \sin (2 \theta)+\alpha^{2} \sin ^{2}(\theta)\right) \\
& =\alpha \sin (2 \theta)+\alpha^{2}\left(\sin ^{2}(\theta)-\frac{1}{2} \sin ^{2}(2 \theta)\right)+O\left(\alpha^{3}\right) \\
& =\alpha \sin (2 \theta)+\frac{1}{4} \alpha^{2} \cos (4 \theta)-\frac{1}{2} \alpha^{2} \cos (2 \theta)+\frac{1}{4} \alpha^{2}+O\left(\alpha^{3}\right) .
\end{aligned}
$$

This lets us write

$$
\log R(n+1)-\log R(n)=\frac{1}{2} a_{n}^{2}+a_{n} X_{n},
$$

and, as in Sect. 6 , use the integration by parts machine to prove

$$
\left(\int\left(\sum_{n=1}^{N} a_{n} X_{n}\right)^{2} g(k) d k\right)^{1 / 2} / \sum a_{n}^{2} \rightarrow 0
$$

and complete the proof as there.

In this case, we don't need to worry about zeros of $\widetilde{W}(k)$ since the analog of $W$ here is $\delta_{n 0}$ and so $\widetilde{W}(k)=1$.

\section{Random Decaying Potentials: The Discrete Case}

In this section, we consider discrete situations where the $V(n)$ are independent random variables of zero mean and decaying variance. The results that imply a.c. spectrum require no regularity in $E\left(V(n)^{2}\right)$, while those for singular spectrum require some kind of regular decay, as we will explain.

The results for a.c. spectrum are so general yet so simple to prove that they are a paradigm of the usefulness of the EFGP transform.

Theorem 8.1. Suppose $V_{\omega}(n)$ are independent random variables with $E\left(V_{\omega}(n)\right)=0$ and

$$
\sum_{n} E\left(V_{\omega}(n)^{2}\right)+E\left(V_{\omega}(n)^{4}\right)<\infty .
$$

Then for a.e. $\omega, h_{\omega}$ has purely a.c. spectrum on $(-2,2)$.

Remarks. 1. For $E\left(V_{\omega}^{2}\right)^{1 / 2} \leq C n^{-\alpha}$ with $V$ bounded and $\alpha>\frac{1}{2}$, we get a.c. spectrum recovering results of Delyon, et al. [7].

2. If the $V_{\omega}(n)$ are uniformly bounded, then $E\left(V_{\omega}(n)^{4}\right) \leq C E\left(V_{\omega}(n)^{2}\right)$ and so (8.1) becomes $\sum_{n} E\left(V_{\omega}(n)^{2}\right)<\infty$; we state the general bound because unbounded $V$ 's are so easy to accommodate.

3. The case $E\left(V_{\omega}(n)^{2}\right)^{1 / 2}=n^{-1 / 2} \log (n)^{-1}$ is of some interest. This sequence is $\ell^{2}$ so if $V$ is bounded, the theorem proves a.c. spectrum. Kotani-Ushiroya [21] cannot handle such borderline cases.

Proof. Fix $\theta_{0}$. Then $R_{\omega}(n)$ and $\theta_{\omega}(n)$ become random variables which are measurable functions of $\left\{V_{\omega}(j)\right\}_{j \leq n-1}$ and so independent of $\left\{V_{\omega}(j)\right\}_{j \geq n}$.

By $(2.12 \mathrm{c})$,

$$
R(n+1)^{4}=R(n)^{4}\left(1+\frac{V_{\omega}(n)}{\sin k} \sin \left(2 \bar{\theta}_{\omega}(n)\right)+O\left(V_{\omega}^{2}+V_{\omega}^{4}\right)\right)
$$

Since $V_{\omega}(n)$ is independent of $\bar{\theta}(n)$ and $R(n)$, we have

$$
E\left(R_{\omega}(n)^{4} V_{\omega}(n) \sin \left(2 \overline{\theta_{\omega}(n)}\right)\right)=E\left(V_{\omega}(n)\right) E\left(R_{\omega}^{4}(n) \sin \left(2 \bar{\theta}_{\omega}(n)\right)\right)=0 .
$$


Using independence to bound $E\left(R(n)^{4} V_{\omega}^{j}\right)$ by $E\left(R(n)^{4}\right) E\left(V_{\omega}^{j}\right)$, we see that

$$
E\left(R_{\omega}(n+1)^{4}\right) \leq\left[1+C E\left(V_{\omega}^{2}(n)+V_{\omega}^{4}(n)\right)\right] E\left(R_{\omega}^{4}(n)\right),
$$

where $C$ is uniformly bounded for $k$ in any $(\alpha, \pi-\alpha)$ with $\alpha>0$. It follows that

$$
E\left(\int_{\alpha}^{\pi-\alpha} R_{\omega}(n, k)^{4} d k\right)<\infty .
$$

By Fatou's lemma, for a.e. $\omega$,

$$
\underline{\lim } \int_{\alpha}^{\pi-\alpha} R_{\omega}(n, k)^{4} d k<\infty,
$$

and by Theorem 1.3, the spectrum is purely a.c. on $(-2 \cos (\alpha), 2 \cos (\alpha))$.

For the case where $\sum_{n=1}^{\infty} E\left(V(n)^{2}\right)=\infty$, we need some regularity of the fall-off. Rather than try to find complicated general conditions, we consider the case where $E\left(V(n)^{2}\right) \sim n^{-2 \alpha}$ with $\alpha \leq \frac{1}{2}$. The same method can handle a case like $E\left(V(n)^{2}\right)=$ $[n \log (n+1)]^{-1}$ (which always has singular continuous spectrum of Hausdorff dimension 1 ) by the kind of arguments we will discuss in the case $\alpha=\frac{1}{2}$; in this case for typical energies $\|T(0, n)\|$ grows like $\log (n)$.

Explicitly, we suppose

(i) $E\left(V_{\omega}(n)^{2}\right)^{1 / 2}=\lambda n^{-\alpha} \quad 0<\alpha \leq \frac{1}{2} ; \lambda>0$,

(ii) $E\left(V_{\omega}(n)\right)=0$,

(iii) For some $\epsilon>0, \sup _{\omega}\left|V_{\omega}(n)\right| \leq C n^{-(2 \alpha / 3)-\epsilon}$,

(iv) $V_{\omega}(n)$ is independent of $\left\{V_{\omega}(j)\right\}_{j=1}^{n-1}$.

Remarks. 1. Think of the case discussed in [26, 7], where $V_{\omega}(n)=n^{-\alpha} X_{n}(\omega)$ with $X_{n}$ identically distributed bounded, independent random variables. If $E(X)=0$ and $X$ is bounded, then (i)-(iv) hold.

2. With some extra effort, we could allow unbounded distributions, and only require that $\lim _{n \rightarrow \infty} n^{+\alpha} E\left(V_{\omega}(n)^{2}\right)^{1 / 2}$ exists and be non-zero.

Theorem 8.2. Suppose (i)-(iv) hold. Fix $k$ in $(0, \pi)$ with $k \neq \frac{\pi}{4}, \frac{2 \pi}{4} \frac{3 \pi}{4}$. Then for a.e. $\omega$,

$$
\lim _{n \rightarrow \infty} \frac{\log \left\|T_{2 \cos (k)}(n, 0)\right\|}{\left(\sum_{j=1}^{n} j^{-2 \alpha}\right)}=\frac{\lambda^{2}}{8 \sin ^{2}(k)} .
$$

Remark. In case $\alpha<\frac{1}{2}$, this says $\|T(n, 0)\| \sim \exp \left(C n^{1-\alpha}\right)$ with $C=\frac{\lambda^{2}}{8(1-2 \alpha) \sin ^{2}(k)}$. If $\alpha=\frac{1}{2}$, this says $\|T\| \sim n^{C}$ with $C=\frac{\lambda^{2}}{8 \sin ^{2}(k)}$.

Proof. By Theorem 2.3, we need only prove this result with $R(n)$ replacing $T$ for each $\theta_{0}$. So fix $k$ and $\theta_{0}$, and let $\theta_{\omega}(n), R_{\omega}(n)$ solve (2.12). By (2.12c),

$$
\log R(n+1)-\log R(n)=\frac{1}{2} \log \left(1+\nu_{k}(n) \sin (2 \bar{\theta}(n))+\nu_{k}(n)^{2} \sin ^{2}(\bar{\theta}(n))\right) .
$$

Since $\sup _{\omega} \nu_{k}(n) \rightarrow 0$ as $n \rightarrow \infty$, we can use

$$
\log (1+x)=x-\frac{x^{2}}{2}+O\left(x^{3}\right) .
$$


We also use

$$
\sin ^{2} \theta-\frac{1}{2} \sin ^{2}(2 \theta)=\frac{1}{4}-\frac{1}{2} \cos (2 \theta)+\frac{1}{4} \cos (4 \theta) .
$$

The net result is

$$
\log R(n)=\frac{1}{8} \sum_{j=1}^{n} \frac{E\left(V_{\omega}(n)^{2}\right)}{\sin ^{2}(k)}+C_{1}+C_{2}+C_{3}+C_{4},
$$

where the corrections have the form

$$
\begin{aligned}
& C_{1}=-\frac{1}{2 \sin (k)} \sum_{j=1}^{n} V_{\omega}(j) \sin \left(2 \bar{\theta}_{\omega}(j)\right) \\
& C_{2}=\frac{1}{2 \sin ^{2}(k)} \sum_{j=1}^{n}\left[V_{\omega}(j)^{2}-E\left(V_{\omega}(j)^{2}\right)\right]\left[\sin ^{2}\left(\bar{\theta}_{\omega}(j)\right)-\frac{1}{2} \sin ^{2}\left(2 \bar{\theta}_{\omega}(j)\right)\right], \\
& C_{3}=\frac{1}{2 \sin ^{2}(k)} \sum_{j=1}^{n} E\left(V_{\omega}(j)^{2}\right)\left[\frac{1}{2} \cos \left(2 \bar{\theta}_{\omega}(j)\right)-\frac{1}{4} \cos \left(4 \bar{\theta}_{\omega}(j)\right)\right] \\
& C_{4}=\sum_{j=1}^{n} O\left(V_{\omega}(j)^{3}+V_{\omega}(j)^{4}\right)
\end{aligned}
$$

The theorem follows if we prove that for each $q=1,2,3,4$ and a.e. $\omega$,

$$
\varlimsup_{n \rightarrow \infty} \frac{\left|C_{q}(\omega)\right|}{\sum_{j=1}^{n} j^{-2 \alpha}}=0 .
$$

Equation (8.4) for $q=4$ is an immediate consequence of hypothesis (iii).

$C_{1}, C_{2}$ clearly have zero expectation values and variances that decay properly for us to hope (8.4) holds; the key to the proof will be a Martingale inequality. $C_{3}$ will depend on the fact that $\cos (\theta)$ has zero average and the slow variation of $E\left(V_{\omega}(n)^{2}\right)$.

We break the proof to present some needed lemmas. For the first two of these lemmas, let $X_{0}, X_{1}, \ldots, X_{N}$ be independent random variables, where $X_{0}$ can be vector valued. Suppose that for $j=1, \ldots, N$,

$$
Z_{j}=X_{j} f_{j}\left(X_{1}, \ldots, X_{j-1} ; X_{0}\right)
$$

with $f_{j}$ a measurable function, and that

$$
E\left(X_{j}\right)=0
$$

The following is a variant of a standard Martingale inequality; we provide a proof for the reader's convenience:

\section{Lemma 8.3.}

$$
E\left(\sup _{n=1,2, \ldots, N}\left|Z_{1}+\cdots+Z_{n}\right| \geq r\right) \leq \frac{1}{r^{2}} E\left(\sum_{j=1}^{N} Z_{j}^{2}\right) .
$$


Proof. Define

$$
Y_{n}=\sum_{j=1}^{n} Z_{j}, \quad Q_{n}=\sum_{j=n+1}^{N} Z_{j}
$$

and let

$$
A_{j}=\left\{\omega|| Y_{1}|\leq r,| Y_{2}|\leq r, \ldots,| Y_{j} \mid>r\right\} .
$$

Then $\chi_{n}$, the characteristic function of $A_{n}$, is a function only of $X_{0}, X_{1}, \ldots, X_{n}$ and thus, if $k>n$,

$$
E\left(Z_{k} Y_{n} \chi_{A_{n}}\right)=E\left(X_{k}\right) E\left(f_{k}\left(X_{1}, \ldots, X_{k-1}, X_{0}\right) Y_{n} \chi_{A_{n}}\right)=0 .
$$

Thus,

$$
E\left(\chi_{n} Y_{n}^{2}\right) \leq E\left(\chi_{n}\left(Y_{n}+Q_{n}\right)^{2}\right),
$$

since the cross term has zero expectation when we expand the square. Thus,

$$
r^{2} \sum_{j=1}^{n} E\left(\chi_{j}\right) \leq \sum_{j=1}^{N} E\left(\chi_{j} Y_{j}^{2}\right) \leq \sum_{j=1}^{N} E\left(\chi_{j} Y_{N}^{2}\right) \leq E\left(Y_{N}^{2}\right)
$$

which is (8.7).

Lemma 8.4. Suppose $E\left(Z_{n}^{2}\right) \leq C n^{-2 \alpha}$. Then for a.e. $\omega$ :

(1) If $\alpha<\frac{1}{2}$ and $\beta>\frac{1}{2}(1-2 \alpha)$, then

$$
\lim _{n \rightarrow \infty}\left|\sum_{j=1}^{n} Z_{j}\right| n^{-\beta}=0
$$

(2) If $\alpha=\frac{1}{2}$ and $\beta>\frac{1}{2}$, then

$$
\lim _{n \rightarrow \infty}\left|\sum_{j=1}^{n} Z_{j}\right|(\log n)^{-\beta}=0 .
$$

(3) If $\alpha>\frac{1}{2}$,

$$
\lim _{n \rightarrow \infty} \sum_{j=1}^{n} Z_{j}=Y_{\infty}
$$

exists, and for any $\beta<\alpha-\frac{1}{2}$,

$$
\lim _{n \rightarrow \infty} n^{+\beta}\left|\sum_{j=n}^{\infty} Z_{j}\right|=0
$$

Remark. Naively, fluctuations should behave as $\left(\sum_{j=1}^{n} j^{-2 \alpha}\right)^{1 / 2}$. This lemma shows they are not much worse. Since we only need that they are small compared to $\sum_{j=1}^{n} j^{-2 \alpha}$, the lemma suffices. 
Proof. (1) Pick $\beta_{1}$ so $\beta>\beta_{1} \geq \frac{1}{2}(1-2 \alpha)$. By Lemma 8.3,

$$
\begin{aligned}
E\left(\sup _{j=1, \ldots, 2^{n-1}}\left|\sum_{k=2^{n-1}+1}^{2^{n-1}+j} Z_{k}\right| \geq 2^{n \beta_{1}}\right) & \leq C 2^{-2 n \beta_{1}} 2^{(n-1)} 2^{-2(n-1) \alpha} \\
& \leq C 2^{-(n-1)\left(2 \alpha+2 \beta_{1}-1\right)}
\end{aligned}
$$

is summable in $n$ by the choice of $\beta_{1}$. Therefore, by the Borel-Cantelli lemma, for a.e. $\omega$, there is an $n_{0}\left(\omega_{0}\right)$ so that the sup inside (8.8) is less than $2^{n \beta_{1}}$ if $n \geq n_{0}$. Let $j$ be larger than $2^{n_{0}-1}$ and pick $n$ so that $2^{n-1}+1 \leq j \leq 2^{n}$. Then

$$
\begin{aligned}
\left|Z_{1}+\cdots+Z_{j}\right| & \leq\left|Z_{1}+\cdots+Z_{2^{n_{0}}}\right|+\sum_{k=1}^{n} 2^{k \beta_{1}} \\
& \leq\left|Z_{1}+\cdots+Z_{2^{n_{0}}}\right|+\frac{2^{n \beta_{1}}}{2^{\beta_{1}}-1} \\
& \leq\left|Z_{1}+\cdots+Z_{2^{n_{0}}}\right|+\frac{2^{\beta_{1}}}{2^{\beta_{1}}-1} j^{\beta_{1}} .
\end{aligned}
$$

Thus, $\overline{\lim } j^{-\beta_{1}}\left|Z_{1}+\cdots+Z_{j}\right|<\infty$. Since $\beta>\beta_{1}$, the limit for $\beta$ is 0 .

(2) Pick $\beta_{1}$ with $\beta>\beta_{1}>\frac{1}{2}$ and define

$$
K_{n}=\left\{\omega\left|\sup _{j=1, \ldots, 2^{n}}\right| \sum_{m=1}^{j} Z_{j} \mid \geq n^{\beta_{1}}\right\} .
$$

Then by Lemma 8.3,

$$
E\left(K_{n}\right) \leq n^{-2 \beta_{1}} \sum_{1}^{2^{n}} \frac{1}{j} \leq n^{-2 \beta_{1}}(1+n \log 2) \leq C n^{1-2 \beta_{1}}
$$

since $\sum_{1}^{k} \frac{1}{j} \leq 1+\log k$.

Pick an integer $m$ so $m\left(2 \beta_{1}-1\right)>1$. Then

$$
\sum_{n=1}^{\infty} E\left(K_{n^{m}}\right)<\infty
$$

So by the Borel-Cantelli lemma, for a.e. $\omega$, there is $n_{0}(\omega)$, so if $n \geq n_{0}$, then $\omega \notin K_{n^{m}}$. If $j>2^{n_{0}^{m}}$, pick $n$ so that

$$
2^{(n-1)^{m}}<j \leq 2^{n^{m}} .
$$

Then

$$
\left|Z_{1}+\cdots+Z_{j}\right| \leq\left(n^{m}\right)^{\beta_{1}} \leq 2^{m \beta_{1}}(n-1)^{m \beta_{1}} \leq 2^{m \beta_{1}}(\log 2)^{-\beta_{1}}(\log j)^{\beta_{1}} .
$$

(3) Pick $\beta_{1}$ so $\beta<\beta_{1}<\alpha-\frac{1}{2}$. Then

$$
\begin{aligned}
E\left(\sup _{j=1, \ldots, 2^{n-1}}\left|\sum_{k=2^{n-1}+1}^{2^{n-1}+j} Z_{k}\right| \geq 2^{-n \beta_{1}}\right) & \leq C 2^{-2 n \beta_{1}} 2^{n-1} 2^{-2(n-1) \alpha} \\
& \leq C 2^{2 \beta_{1}} 2^{-2(n-1)\left[\alpha-1 / 2-\beta_{1}\right]}
\end{aligned}
$$


is summable. Thus, for a.e. $\omega$, there is an $n_{0}(\omega)$ so that for $n \geq n_{0}(\omega)$, the sup is bounded by $2^{-n \beta_{1}}$. Thus, if $j_{1} \geq j_{2} \geq 2^{n_{2}-1} \geq 2^{n_{0}-1}$,

$$
\left|\sum_{k=j_{1}}^{j_{2}} Z_{k}\right| \leq \sum_{n=n_{2}}^{\infty} 2^{-n \beta_{1}} \rightarrow 0
$$

as $n_{2} \rightarrow \infty$. So the sum is convergent (i.e., the partial sums are Cauchy). Moreover, if $j \geq 2^{n_{0}-1}$ and $n$ is picked so $2^{n-1} \leq j \leq 2^{n}$, then

$$
\left|\sum_{k=j}^{\infty} Z_{k}\right| \leq \sum_{m=n}^{\infty} 2^{-m \beta_{1}}=\frac{2^{-n \beta_{1}}}{1-2^{-\beta_{1}}} \leq \frac{j^{-\beta_{1}}}{1-2^{-\beta_{1}}},
$$

and thus, if we multiply by $j^{\beta}$, the limit is 0 .

Lemma 8.5. Suppose that $k \in \mathbb{R}$ is not in $\mathbb{Z} \pi$. Then there exist integers $q_{\ell} \rightarrow \infty$ so that for any $\theta_{0}, \ldots, \theta_{q_{\ell}}$,

$$
\left|\sum_{j=1}^{q_{\ell}} \cos \left(\theta_{j}\right)\right| \leq 1+\sum_{j=1}^{q_{\ell}}\left|\theta_{j}-\theta_{0}-k j\right| .
$$

Remark. In essence, we show $\left|\sum_{j=1}^{q} \cos \left(\theta_{0}+k j\right)\right| \leq 1$ a stronger result than the ergodic theory result that $\left|\frac{1}{q} \sum_{j=1}^{q} \cos \left(\theta_{0}+k j\right)\right| \rightarrow 0$. The weaker ergodic theory result suffices for our application, but the proof of this lemma is easy so we give it.

Proof. By general number theory considerations [14], we can find $p_{\ell}, q_{\ell}$ so that

$$
\left|k-\frac{\pi p_{\ell}}{q_{\ell}}\right| \leq \frac{1}{q_{\ell}^{2}}
$$

and $p_{\ell} / q_{\ell} \notin \mathbb{Z}$ if $k \notin \mathbb{Z} \pi$. For any $p / q \notin \mathbb{Z}$ and any $\theta_{0}$,

$$
\sum_{j=1}^{q} \cos \left(\theta_{0}+\frac{j p \pi}{q}\right)=0 .
$$

Thus

$$
\begin{aligned}
\left|\sum_{j=1}^{q_{\ell}} \cos \left(\theta_{j}\right)\right| & =\left|\sum_{j=1}^{q_{\ell}} \cos \left(\theta_{j}\right)-\cos \left(\theta_{0}+\frac{j p_{\ell} \pi}{q_{\ell}}\right)\right| \\
& \leq \sum_{j=1}^{q_{\ell}}\left|\left(\theta_{j}-\theta_{0}-\frac{j p_{\ell} \pi}{q_{\ell}}\right)\right| \\
& \leq \sum_{j=1}^{q_{\ell}}\left|\theta_{j}-\theta_{0}-k j\right|+\sum_{j=1}^{q_{\ell}} j\left|k-\frac{\pi p_{\ell}}{q_{\ell}}\right| \\
& \leq \frac{q_{\ell}\left(q_{\ell}+1\right)}{2 q_{\ell}^{2}}+\sum_{j=1}^{q_{\ell}}\left|\theta_{j}-\theta_{0}-k j\right| .
\end{aligned}
$$


Conclusion of the Proof of Theorem 8.2. We need to verify (8.4) for $q=1,2,3$. $V_{\omega}(n) \sin \left(2 \bar{\theta}_{\omega}(n)\right) \equiv Z_{n}$ has the form (8.5) and $E\left(Z_{n}^{2}\right) \leq C n^{-2 \alpha}$, so by Lemma 8.4, for a.e. $\omega$,

$$
\left|C_{1}(\omega)\right|=o\left(\sum_{j=1}^{n} j^{-2 \alpha}\right) .
$$

$\left[V_{\omega}(n)^{2}-E\left(V_{\omega}(n)^{2}\right)\right]\left[\sin ^{2}(\bar{\theta})-\frac{1}{2} \sin ^{2}(2 \theta)\right]$ also has the form of (8.4) since $E\left(V_{\omega}^{2}-\right.$ $\left.E\left(V_{\omega}^{2}\right)\right)=0$. Since $V$ is bounded,

$$
E\left(\left(V^{2}-E\left(V^{2}\right)\right)^{2}\right) \leq C E\left(V^{2}\right) .
$$

Thus, for a.e. $\omega$,

$$
\left|C_{2}(\omega)\right|=o\left(\sum_{j=1}^{n} j^{-2 \alpha}\right)
$$

also.

Finally, we will show

$$
\sum_{j=1}^{n} j^{-2 \alpha} \cos \left(4 \bar{\theta}_{\omega}(j)\right)=o\left(\sum_{j=1}^{n} j^{-2 \alpha}\right),
$$

which proves (8.4) for $q=3$. By hypothesis on $k, 4 k \notin \mathbb{Z} \pi$ so Lemma 8.5 applies. Let $q_{\ell}$ be as in that lemma. Note next that by hypothesis (iii) and Proposition 2.4 for $j$ large,

$$
\left|\theta_{\omega}(j+1)-\theta_{\omega}(j)-k\right| \leq C_{0} j^{-2 \alpha / 3}
$$

Pick $n_{0}$ so

$$
n_{0} \geq q_{\ell}^{2}
$$

and

$$
4 C_{0} n_{0}^{-2 \alpha / 3} \leq q_{\ell}^{-2}
$$

Suppose $N=n_{0}+K q_{\ell}$. Then

$$
\begin{aligned}
\left|\sum_{j=n_{0}+1}^{N} j^{-2 \alpha} \cos \left(4 \theta_{\omega}(j)\right)\right| & =\left|\sum_{m=0}^{K} \sum_{j=1}^{q_{\ell}}\left(n_{0}+m q_{\ell}+j\right)^{-2 \alpha} \cos \left(4 \theta_{\omega}\left(m q_{\ell}+j\right)\right)\right| \\
& =A_{1}+A_{2},
\end{aligned}
$$

where $A_{1}$ is what we get by replacing $\left(n_{0}+k q_{\ell}+j\right)^{-2 \alpha}$ by $\left(n_{0}+k q_{\ell}\right)^{-2 \alpha}$ and $A_{2}$ is the difference. By Lemma 8.5, (8.10), and (8.12),

$$
A_{1} \leq \sum_{k=0}^{K}\left(n_{0}+k q_{\ell}\right)^{-2 \alpha}[1+1],
$$

while using

$$
\left|\left(n_{0}+k q_{\ell}+j\right)^{-2 \alpha}-\left(n_{0}+k q_{\ell}\right)^{-2 \alpha}\right| \leq\left(n_{0}+k q_{\ell}\right)^{-2 \alpha} j n_{0}^{-1}
$$

and (8.11), 


$$
A_{2} \leq \sum_{k=0}^{K}\left(n_{0}+k q_{\ell}\right)^{-2 \alpha} q_{\ell}^{2} n_{0}^{-1} \leq \sum_{k=0}^{K}\left(n_{0}+k q q^{-2 \alpha} .\right.
$$

Thus for any $N$,

$$
\left|\sum_{1}^{N} j^{-2 \alpha} \cos \left(4 \theta_{\omega}(j)\right)\right| \leq C_{\ell}+3 q_{\ell}^{-\alpha} \sum_{j=1}^{N} j^{-2 \alpha},
$$

and so

$$
\varlimsup_{N \rightarrow \infty}\left(\sum_{j=1}^{N} j^{-2 \alpha}\right)^{-1}\left|\sum_{1}^{N} j^{-2 \alpha} \cos \left(4 \theta_{\omega}(j)\right)\right| \leq 3 q_{\ell}^{-\alpha}
$$

uniformly in $\omega$. Since we can take $q_{\ell} \rightarrow \infty$ by Lemma 8.5 , the $\varlimsup$ im is 0 .

Theorem 8.6. Suppose that (i)-(iv) hold with $\alpha<\frac{1}{2}$ but we consider $V(1)$ as a continuous parameter. Then for a.e. $\omega$ :

(1) For a dense $G_{\delta}$ of values of $V(1), H_{\omega}$ has purely singular continuous spectrum in $(-2,2)$.

(2) For Lebesgue a.e. value of $V(1), H_{\omega}$ has dense pure point spectrum in $(-2,2)$ and the eigenfunctions obey $H_{\omega} u=2 \cos \left(k_{m}\right) u$ with

$$
\lim _{n \rightarrow \infty} \frac{\log \left(\left|u(n)^{2}+u(n+1)^{2}\right|^{1 / 2}\right)}{|n|^{1-2 \alpha}}=-\frac{(1-2 \alpha) \lambda^{2}}{8 \sin ^{2}\left(k_{m}\right)} .
$$

If we consider a whole-line problem with independent $V_{\omega}(n)$, where both $\left\{V_{\omega}(n)\right\}_{n=1}^{\infty}$ and $\tilde{V}_{\omega}(n) \equiv V_{\omega}(-n), n=1,2, \ldots$ obey hypotheses (i)-(iv) and $V_{\omega}(0)$ has a purely a.c. density, then for a.e. $\omega, H_{\omega}$ has dense pure point spectrum in $(-2,2)$ and $(8.13)$ holds as $|n| \rightarrow \infty$.

Remark. This strengthens the result originally proven in [31] and improved in [7] in two ways. First, we get the explicit constant in (8.13). Second, we only require one $V_{\omega}(\cdot)$ to have an a.e. distribution.

Proof. By Theorem 8.2 and Fubini's theorem for a.e. $\omega$, we have for a.e. $k \in(0, \pi)$,

$$
\lim _{n \rightarrow \infty} \frac{\log \|T(n)\|}{n^{1-2 \alpha}}=\frac{(1-2 \alpha) \lambda^{2}}{8 \sin ^{2}(k)} .
$$

Thus by Theorem 8.3 of [22], there is an $L^{2}$-solution obeying (8.13). The theorem follows from general principles on rank one perturbations $[12,4,5,28]$.

The case $\alpha=\frac{1}{2}$ has an extra subtlety we will need to deal with, using an argument modeled on Kotani-Ushiroya [21]. The following replaces an explicit but complex formula they use for the projection onto a decaying solution (and fills in a gap in their argument):

Lemma 8.7. Let $u_{\theta}=(\cos \theta, \sin \theta)$ in $\mathbb{R}^{2}$. For any unimodular matrix $A$ with $\|A\|>$ 1 , let $\theta(A)$ be the unique $\theta \in\left(-\frac{\pi}{2}, \frac{\pi}{2}\right]$ with $\left\|A u_{\theta}\right\|=\|A\|^{-1}$. Define $\rho(A)=$ $\left\|A u_{0}\right\| /\left\|A u_{\pi / 2}\right\|$. Let $A_{n}$ be a sequence of unimodular matrices with $\left\|A_{n}\right\| \rightarrow \infty$ and $\left\|A_{n+1} A_{n}^{-1}\right\| /\left\|A_{n}\right\|\left\|A_{n+1}\right\| \rightarrow 0$ as $n \rightarrow \infty$. Let $\rho_{n}=\rho\left(A_{n}\right), \theta_{n}=\theta\left(A_{n}\right)$. Then: 
(i) $\theta_{n}$ has a limit $\theta_{\infty}$ if and only if $\lim _{n \rightarrow \infty} \rho_{n} \equiv \rho_{\infty}$ exists $\left(\rho_{\infty}=\infty\right.$ is allowed, but then we only have $\left|\theta_{n}\right| \rightarrow \frac{\pi}{2}$ ).

(ii) Suppose $\theta_{n}$ has a limit $\theta_{\infty} \neq 0, \frac{\pi}{2}$ (equivalently, $\rho_{\infty} \neq 0, \infty$ ). Then

$$
\lim _{n \rightarrow \infty} \frac{\log \left\|A_{n} u_{\infty}\right\|}{\log \left\|A_{n}\right\|}=-1
$$

if and only if

$$
\varlimsup_{n \rightarrow \infty} \frac{\log \left|\rho_{n}-\rho_{\infty}\right|}{\log \left\|A_{n}\right\|} \leq-2
$$

Remark. Consider

$$
A_{n}=\left(\begin{array}{cc}
\cosh (n) & (-1)^{n} \sinh (n) \\
(-1)^{n} \sinh (n) & \cosh (n)
\end{array}\right)
$$

Then $\rho\left(A_{n}\right) \equiv 1$ and $\left\|A_{n}\right\| \rightarrow \infty$ but $\theta_{n}=(-1)^{n+1}\left(\frac{\pi}{4}\right)$ does not have a limit. This shows that the condition $\left\|A_{n+1} A_{n}^{-1}\right\| /\left\|A_{n}\right\|\left\|A_{n+1}\right\| \rightarrow 0$ is required. Indeed, in this case that limit is 1 . Kotani-Ushiroya miss this issue.

Proof. (i) Note first that

$$
\left\|A_{n} u_{\theta}\right\|^{2}=\left\|A_{n}\right\|^{2} \sin ^{2}\left(\theta-\theta_{n}\right)+\left\|A_{n}\right\|^{-2} \cos ^{2}\left(\theta-\theta_{n}\right) .
$$

Thus,

$$
\rho_{n}=\frac{\tan ^{2}\left(\theta_{n}\right)+\left\|A_{n}\right\|^{-4}}{1+\left\|A_{n}\right\|^{-4} \tan ^{2}\left(\theta_{n}\right)} .
$$

It follows that $\rho_{n}$ has a finite limit $\rho_{\infty}$ if $\tan ^{2}\left(\theta_{n}\right)$ has a finite limit. By writing

$$
\rho_{n}^{-1}=\frac{\cot ^{2}\left(\theta_{n}\right)+\left\|A_{n}\right\|^{-4}}{1+\left\|A_{n}\right\|^{-4} \cot ^{2}\left(\theta_{n}\right)},
$$

this is true also for $\rho_{n} \rightarrow \infty$ and $\tan ^{2}\left(\theta_{n}\right) \rightarrow \infty$.

Pick $\eta \in\left[0, \frac{\pi}{2}\right]$ so $\tan ^{2}\left(\theta_{n}\right) \rightarrow \tan ^{2}(\eta)$. If $\eta=0$, then $\theta_{n} \rightarrow 0$, and if $\eta \rightarrow \frac{\pi}{2}$, then $\left|\theta_{n}\right| \rightarrow \frac{\pi}{2}$ because of the continuity of $\tan (\theta)$ on $\left[-\frac{\pi}{2}, \frac{\pi}{2}\right]$. If $0<\eta<\frac{\pi}{2}$, we only have $\left|\theta_{n}\right| \rightarrow \eta$ and have to worry about the sign (see the remark above).

In (8.16), take $\theta=\theta_{n+1}$ and see that

$$
\begin{aligned}
\sin ^{2}\left(\theta_{n+1}-\theta_{n}\right) & \leq\left\|A_{n}\right\|^{-2}\left\|A_{n} A_{n+1}^{-1}\right\|^{2}\left\|A_{n+1} u_{\theta_{n+1}}\right\|^{2} \\
& =\left\|A_{n}\right\|^{-2}\left\|A_{n+1}\right\|^{-2}\left\|A_{n+1} A_{n}^{-1}\right\|^{2}
\end{aligned}
$$

since $A_{n} A_{n+1}^{-1}$ is unimodular, and thus $\left\|A_{n} A_{n+1}^{-1}\right\|=\left\|A_{n+1} A_{n}^{-1}\right\|$. Thus by hypothesis,

$$
\sin ^{2}\left(\theta_{n+1}-\theta_{n}\right) \rightarrow 0
$$

This, together with $\left|\theta_{n}\right| \rightarrow \eta \in\left(0, \frac{\pi}{2}\right)$, implies that $\theta_{n}$ has a limit.

(ii) By (8.16), we have that (8.14) holds if and only if

$$
\lim _{n \rightarrow \infty} \frac{\log \left|\theta_{n}-\theta_{\infty}\right|}{\log \left\|A_{n}\right\|} \leq-2 .
$$

Since $\theta_{\infty} \neq 0, \pi$, this is true if and only if 


$$
\lim _{n \rightarrow \infty} \frac{\log \left|\tan ^{2}\left(\theta_{n}\right)-\tan ^{2}\left(\theta_{\infty}\right)\right|}{\log \left\|A_{n}\right\|} \leq-2 .
$$

By (8.17) and $\theta_{\infty} \neq \pi$,

$$
|| \tan ^{2}\left(\theta_{n}\right)-\tan ^{2}\left(\theta_{\infty}\right)|-| \rho_{n}-\rho_{\infty}\|\leq C\| A_{n} \|^{-4} .
$$

Thus, (8.18) holds if and only if (8.15) holds.

Lemma 8.8. Suppose the hypotheses of Theorem 8.2 hold with $\alpha=\frac{1}{2}$ and $k \neq \frac{\pi}{4}, \frac{2 \pi}{4}, \frac{3 \pi}{4}$ is fixed. Then for a.e. $\omega$, there exists an initial condition $u_{\theta(\omega)}$ so that

$$
\lim _{n \rightarrow \infty} \frac{\log \left\|T_{2 \cos (k)}(n, 0) u_{\theta(\omega)}\right\|}{\log (n)}=-\frac{\lambda^{2}}{8 \sin ^{2}(k)} .
$$

Remark. As noted in [22] (and gotten incorrectly in [21]), Ruelle's deterministic argument doesn't ever suffice in this $\|T\| \sim n^{\gamma}$ case. If $A_{n}$ is a sequence of unimodular matrices with $\lim _{n \rightarrow \infty} \log \left\|A_{n}\right\| / \log (n)=\gamma$, then [22] has explicit examples (even coming from deterministic Schrödinger operators) for each $\gamma>\frac{1}{2}$ where the decaying solution only obeys $\lim _{n \rightarrow \infty} \log \left\|A_{n} u_{\infty}\right\| / \log (n)=-\gamma+1$. It also appears one needs $\gamma>\frac{3}{2}$ to be sure of the existence of decaying solutions. But following [21], the probabilistic argument here can replace Ruelle's argument.

Proof. Let $\beta=\frac{\lambda^{2}}{8 \sin ^{2}(k)}$. Let $R_{1}(n)$ and $R_{2}(n)$ be the $R$ 's associated to $\theta=0$ and $\theta=\frac{1}{2}$. By the proof of Theorem 8.2 for a.e. $\omega$,

$$
\lim _{n \rightarrow \infty} \frac{\log \left\|R_{i}(n)\right\|}{\log (n)}=\beta .
$$

Let $\theta_{i}(n)$ be the corresponding EFGP angles. By (2.7),

$$
R_{1}(n) R_{2}(n) \sin \left(\theta_{1}(n)-\theta_{2}(n)\right)=\sin (k)\left[u_{1}(n) u_{2}(n-1)-u_{1}(n-1) u_{2}(n)\right]=-1
$$

(by the initial conditions $R_{1}(1)=R_{2}(1)=0, \theta_{1}(1)=0, \theta_{2}(1)=\frac{\pi}{2}$ ) and constancy of the Wronskian. Thus by (8.19) for a.e. $\omega$,

$$
\lim _{n \rightarrow \infty} \frac{\log \left|\theta_{1}(n)-\theta_{2}(n)\right|}{\log (n)}=-2 \beta .
$$

Let $\rho_{n}=\frac{R_{1}(n)}{R_{2}(n)}$. Then by $(2.12 \mathrm{c})$,

$$
L_{\omega}(n) \equiv[\log \rho(n+1)-\log \rho(n)]=\log \left(1+A_{1}(n)\right)-\log \left(1+A_{2}(n)\right),
$$

where

$$
A_{i}(n)=-\frac{V_{\omega}(n)}{\sin (k)} \sin \left(2 \theta_{i, \omega}(n)\right)+\frac{V_{\omega}(n)^{2}}{\sin ^{2}(k)} \sin ^{2}\left(\theta_{i, \omega}(n)\right) .
$$

Define

$$
F(a, \theta)=\log \left(1-a \sin (2 \theta)+a^{2} \sin ^{2}(\theta)\right) .
$$

By a finite Taylor expansion,

$$
F(a, \theta)=\sum_{j=1}^{J-1} a^{j} P_{j}(\theta)+O\left(a^{J}\right)
$$


with $P_{1}(\theta)=\sin (2 \theta)$ and the $P^{\text {'s, }} C^{\infty}$ in $\theta$. Fix $\epsilon>0$ so for $n$ large, use (8.20) to see that $\left|\theta_{1}-\theta_{2}\right|=o\left(n^{-2 \beta+\epsilon}\right)$. Choosing $J$ so $n^{-J / 3}=o\left(n^{-2 \beta-1}\right)$, we see that

$$
L_{\omega}(n)=-\frac{V_{\omega}(n)}{\sin (k)}\left[\sin \left(2 \theta_{1}(n)\right)-\sin \left(2 \theta_{2}(n)\right)\right]+O\left(n^{-2 \beta-1+\epsilon}\right) .
$$

Since $\theta_{j}(n)$ depend only on $\left\{V_{\omega}(k)\right\}_{k \leq n-1}$, we can apply part (3) of Lemma 8.4 (with $2 \alpha=1+2 \beta-\epsilon)$ to see that for a.e. $\omega$,

$$
\lim _{N \rightarrow \infty} \sum_{1}^{N} L_{\omega}(n)
$$

exists and

$$
\left|\sum_{N}^{\infty} L_{\omega}(n)\right| \leq C_{\omega} N^{-2 \beta+\epsilon} .
$$

By (8.21), $\lim \frac{R_{1}(n)}{R_{2}(n)} \equiv \rho_{\infty}$ exists and is different from 0 and $\infty$. Moreover, by (8.22),

$$
\varlimsup \frac{\log |\rho(n)-\rho(\infty)|}{\log (n)} \leq-2 \beta .
$$

Lemma 8.7 completes the proof.

Theorem 8.9. Suppose (i)-(iv) hold with $\alpha=\frac{1}{2}$. Then,

(1) For a.e. $\omega$, the essential spectrum of $H_{\omega}$ is $[-2,2]$ and the absolutely continuous spectrum of $H_{\omega}$ is empty.

(2) If $|\lambda| \geq 2$ and $V_{\omega}(1)$ has an absolutely continuous distribution, then for a.e. $\omega, H_{\omega}$ has dense point spectrum and only dense point spectrum in $(-2,2)$.

(3) If $|\lambda|<2$ and $V_{\omega}(1)$ has an absolutely continuous distribution, then for a.e. $\omega, H_{\omega}$ has purely singular continuous spectrum in $\left\{E|| E \mid<\left(4-\lambda^{2}\right)^{1 / 2}\right\}$ and only dense pure point spectrum in $\left\{E\left|\left(4-\lambda^{2}\right)^{1 / 2}<\right| E \mid<2\right\}$.

In either case (2) or (3), in the region of point spectrum, there are almost surely eigenvectors of power decay $n^{-\beta}$ with

$$
\beta=\frac{\lambda^{2}}{8-2 E^{2}} .
$$

Remark. This theorem extends results of Delyon, et al. [7], Delyon [6], and KotaniUshiroya [21]. In particular, [7] conjectured that there is a region of point spectrum near $E= \pm 2$ no matter how small $\lambda$ is.

Proof. By Theorem 8.2, $\lim _{n \rightarrow \infty}\left\|T_{\omega}(0, n)\right\|=\infty$ for a.e. $E$ for a.e $\omega$, so by Theorem 1.1, we conclude (3). By Lemma 8.8, for a.e. pairs $(\omega, E)$, there is a unique decaying solution with rate of decay $n^{-\beta}$ with $\beta=\frac{\lambda^{2}}{8-2 E^{2}}$. If $\beta>\frac{1}{2}$, this is $\ell^{2}$ and we have potential point spectrum. If $\beta<\frac{1}{2}$, there is no $\ell^{2}$ solution. The general theory of rank one perturbations ([32, 5]) then yields (2) and (3).

We can compute the precise Hausdorff dimension of the singular continuous spectral measures in this case: 
Theorem 8.10. Fix $\lambda<2$ and a model obeying (i)-(iv) with $\alpha=\frac{1}{2}$. In the region $|E| \leq\left(4-\lambda^{2}\right)^{1 / 2}$, define

$$
d(E, \lambda)=\frac{4-E^{2}-\lambda^{2}}{4-E^{2}}
$$

Suppose $V_{\omega}(1)$ has an absolutely continuous density. Then for a.e. $\omega$, the spectral measure, $\mu$, has dimension $d(E, \lambda)$ at $E$ in the sense that for any $\epsilon$, there is a $\delta$ so that $\mu(A)=0$ if $A$ is a subset of $(E-\delta, E+\delta)$ of Hausdorff dimenion less than $(d-\epsilon)$, and there is a subset $B$ of Hausdorff dimension less than $(d+\epsilon)$, so $\mu((E-\delta, E+\delta) \backslash B)=0$.

Proof. Let $\|u\|_{L}=\left(\sum_{j=1}^{L} u(j)^{2}\right)^{1 / 2}$. By the general theory of rank one perturbations, Theorem 8.2, Lemma 8.8, and the assumption of $V_{\omega}(1)$ for a.e. $\omega, \mu$ is supported on the set of energies where most solutions grow as $n^{\beta}$ and one decays as $n^{-\beta}$, where $\beta(E, \lambda)$ is given by (8.23). The hypothesis for singular spectrum is precisely $\beta<\frac{1}{2}$.

Since $\beta<\frac{1}{2},\left\|u_{1}\right\|_{L} \sim L^{-\beta} L^{1 / 2}$ while $\left\|u_{2}\right\|_{L} \sim L^{\beta} L^{1 / 2}$, where $a \sim b$ is shorthand for $\lim \frac{\log (a)}{\log (b)}=1$. The Jitomirskaya-Last version [16, 17] of the Gilbert-Pearson [11] theory says that the Borel transform of the spectral measure is supported on the set of E's, where

$$
|m(E+i \epsilon)| \sim \frac{\left\|u_{2}\right\|_{L}}{\left\|u_{1}\right\|_{L}},
$$

and $E$ is given by

$$
\left\|u_{1}\right\|_{L}\left\|u_{2}\right\|_{L}=\frac{1}{2 \epsilon}
$$

(the $\sim$ in $(8.26)$ holds in the strong sense that the ratio lies in the interval $(5-\sqrt{24}, 5+$ $\sqrt{24})$ ). Thus, $\epsilon \sim L^{-1}$ and (8.26) says that

$$
|m(E+i \epsilon)| \sim \epsilon^{-2 \beta} .
$$

Since $\beta$ is continuous, the theory in [3] then says that the local dimension is given by $1-2 \beta$ as claimed.

\section{Random Decaying Potentials: The Continuum Case}

Having done the discrete random case, we will only sketch the continuum case. We will specialize to a situation where $\{V(x)\}_{n \leq x<n+1}$ are independent for different $n$ 's. Using ideas from [21], one can presumably use Martingale methods to control asymptotically independent situations.

Theorem 9.1. Let $\left\{V_{\omega}(x)\right\}_{0 \leq x<\infty}$ be a family of random variables and let

$$
a_{n}(\omega)=\int_{n}^{n+1}\left|V_{\omega}(y)\right| d y
$$

Suppose

(i) $E\left(V_{\omega}(x)\right)=0$ for each $x$,

(ii) $\sum_{n} E\left(a_{n}^{2} e^{C a_{n}}\right)<\infty$ for all $C>0$,

(iii) $\{V(x)\}_{n \leq x<n+1}$ are independent for different $n$ 's. 
Then for a.e. $\omega,-\frac{d^{2}}{d x^{2}}+V_{\omega}(x)$ on $L^{2}(0, \infty)$ has purely absolutely continuous spectrum on $(0, \infty)$ for any boundary condition.

Remarks. 1. Our methods imply for all $E>0$ and a.e. $\omega, T_{E}(n)$ is bounded, and that implies $-\frac{d^{2}}{d x^{2}}+V_{\omega}(x)$ is limit point at infinity, so we need not worry about self-adjointness issues.

2. A simple example where (ii) holds is if $\sup _{\omega, x}\left|V_{\omega}(x)\right|<\infty$ and $E\left(\int_{0}^{\infty} V(y)^{2} d y\right)$ $<\infty$.

Proof. By (2.4),

$$
R^{4}(n+1)=R^{4}(n) \exp \left(B_{n}(\omega)\right)
$$

where

$$
B_{n}(\omega)=\frac{2}{k} \int_{n}^{n+1} V_{\omega}(x) \sin \left(2 \theta_{\omega}(x)\right) d x .
$$

By (2.3),

$$
\left|\theta_{\omega}(x)-\theta_{\omega}(n)-k(x-n)\right| \leq \frac{2}{k} a_{n}(\omega)
$$

Using

we obtain from (9.2)-(9.4),

$$
\left|e^{x}-1-x\right| \leq \frac{1}{2} x^{2} e^{x}
$$

$$
\begin{array}{r}
R^{4}(n+1) \leq R^{4}(n)\left(1+C a_{n}^{2} e^{C a_{n}}\right)+Q_{n}, \\
Q_{n}=\frac{2}{k} R^{4}(n) \int_{n}^{n+1} V_{\omega}(x) \sin \left(2 \theta_{\omega}(n)+2 k(x-n)\right) d x
\end{array}
$$

for some constant $C$ uniformly bounded for $k$ in any compact of $(0, \infty)$.

Since $V_{\omega}(x)$ is independent of $\left\{V_{\omega}(y)\right\}_{y \leq n}$, it is independent of $R(n)$ and $\theta_{\omega}(n)$, and so $E\left(Q_{n}\right)=0$.

Moreover, $a_{n}$ is independent of $R(n)$, so (9.5) implies that

$$
E\left(R^{4}(n+1)\right) \leq E\left(R^{4}(n)\right) E\left(1+C a_{n}^{2} e^{C a_{n}}\right) .
$$

By condition (ii), we see that

$$
\varlimsup_{n \rightarrow \infty} E\left(R^{4}(n)\right)<\infty
$$

with bounds uniform in $k$ on compacts of $(0, \infty)$. Thus by Fatou's lemma, for a.e. $\omega$, $\underline{\lim } \int_{a}^{b} R_{\omega}^{4}(n, k) d k<\infty$ and so the spectrum is purely absolutely continuous by Theorem 1.3.

Theorem 9.2. Let $f$ be supported on $(0,1)$ and let

$$
V_{\omega}(x)=\sum_{n=0}^{\infty}(n+1)^{-\alpha} X_{n}(\omega) f(x-n),
$$

where $\left\{X_{n}(\omega)\right\}$ are independent, identically distributed bounded variables of mean zero and $0<\alpha \leq \frac{1}{2}$. Then for $4 k \notin \mathbb{Z} \pi$,

$$
\lim _{n \rightarrow \infty} \frac{\log \|T(n, 0)\|}{\sum_{j=1}^{n} j^{-\alpha}}=\frac{E\left(X_{n}^{2}\right)}{8 k^{2}}\left|\int_{0}^{1} f(y) e^{i k y} d y\right|^{2} .
$$


Remarks. 1. This implies pure point spectrum for a.e. $\omega$ if $\alpha<\frac{1}{2}$.

2. If $\alpha=\frac{1}{2}$, we get singular continuous spectrum for large $E$ and pure point spectrum for small $E$ (assuming $\int_{0}^{1} f(y) d y \neq 0$ or $\int_{0}^{1} y f(y) d y \neq 0$ ) and no a.c. spectrum.

Sketch. Define $\theta_{n}(y)=\theta(n)+k y$ and

$$
\delta \theta_{n}(y)=-(n+1)^{-\alpha} X_{n} \int_{0}^{y} f(y) \sin ^{2}\left(\theta_{n}(y)\right) d y .
$$

By (2.3),

$$
\left|\theta_{n}(n+y)-\theta_{n}(y)-\delta \theta_{n}(y)\right|=O\left(n^{-2 \alpha}\right)
$$

for $y \in(0,1)$.

Plugging this into (2.4), we find that

$$
\log R(n+1)-\log R(n)=Y_{n}^{(1)}+Y_{n}^{(2)}+O\left(n^{-2 \alpha}\right),
$$

where

$$
Y_{n}^{(1)}=\frac{(n+1)^{-\alpha} X_{n}(\omega)}{2 k} \int_{0}^{1} f(y) \sin \left(2 \theta_{n}(y)\right) d y
$$

and

$$
Y_{n}^{(2)}=\frac{(n+1)^{-\alpha} X_{n}(\omega)}{2 k} \int_{0}^{1} 2 f(y) \cos \left(2 \theta_{n}(y)\right)\left(\delta \theta_{n}\right)(y) d y .
$$

By using Lemmas 8.2 and 8.3, one sees that

$$
\left(\sum_{j=0}^{n}(j+1)^{-2 \alpha}\right)\left|\sum_{j=0}^{n} Y_{j}^{(1)}\right| \rightarrow 0
$$

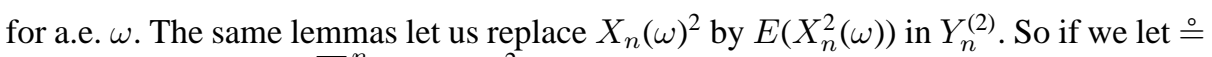
indicate equal up to $o\left(\sum_{j=0}^{n}(j+1)^{-2 \alpha}\right)$ terms, we see that

$$
\log R(n) \stackrel{\circ}{=} \sum_{j=0}^{n-1}\left(Y_{n}^{(3)}+Y_{n}^{(4)}\right)
$$

where we use $\sin ^{2}\left(\theta_{n}(y)\right)=\frac{1}{2}-\frac{1}{2} \cos \left(2 \theta_{n}(y)\right)$ and let $Y_{n}^{(3)}$ indicate the $-\frac{1}{2} \cos (2 \theta)$ terms and $Y_{n}^{(4)}$ the $\frac{1}{2}$ terms. By an argument analogous to the one in the proof of Theorem 9.2 that used Lemma $8.5, \sum Y_{n}^{(4)} \stackrel{\circ}{=} 0$ because $k \notin \mathbb{Z} \pi$.

As in (6.5), we get

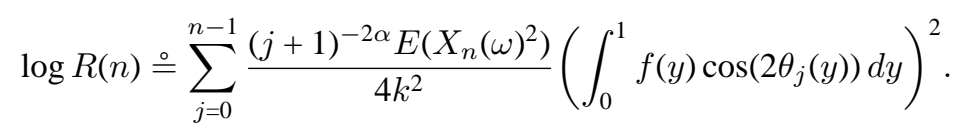

As in the proof of Lemma 6.2, this last square is

$$
\frac{1}{2}\left|\int_{0}^{1} f(y) e^{i k y}\right|^{2}
$$

plus a term that has $\cos \left(4 \theta_{j}(y)\right)$, which we can handle using Lemma 8.5. 


\section{References}

1. Carmona, R.: One-dimensional Schrödinger operators with random or deterministic potentials, New spectral types. J. Funct. Anal. 51, 229-258 (1983)

2. Chulaevsky, V. and Spencer, T.: Positive Lyapunov exponents for a class of deterministic potentials. Commun. Math. Phys. 168, 455-466 (1995)

3. del Rio, R., Jitomirskaya, S., Last, Y. and Simon,B.: Operators with singular continuous spectrum, IV. Hausdorff dimensions, rank one perturbations, and localization. J. d'Analyse Math. 69, 153-200 (1996)

4. del Rio, R., Makarov, N. and Simon, B.: Operators with singular continuous spectrum, II. Rank one operators. Commun. Math. Phys. 165, 59-67 (1994)

5. del Rio, R., Simon, B. and Stolz, G.: Stability of spectral types for Sturm-Liouville operators. Math. Research Lett. 1, 437-450 (1994)

6. Delyon, F.: Apparition of purely singular continuous spectrum in a class of random Schrödinger operators. J. Statist. Phys. 40, 621-630 (1985)

7. Delyon, F., Simon, B. and Souillard, B.: From power pure point to continuous spectrum in disordered systems. Ann. Inst. H. Poincaré 42, 283-309 (1985)

8. Eastham, M.S.P. and Kalf, H.: Schrödinger-type Operators With Continuous Spectra. London: Pitman Books, 1982

9. Eggarter, T.: Some exact results on electron energy levels in certain one-dimensional random potentials. Phys. Rev. B5, 3863-3865 (1972)

10. Gilbert, D.J.: On subordinacy and analysis of the spectrum of Schrödinger operators with two singular endpoints. Proc. Roy. Soc. Edin. 112A, 213-229 (1989)

11. Gilbert, D.J. and Pearson, D.B.: On subordinacy and analysis of the spectrum of one-dimensional Schrödinger operators. J. Math. Anal. 128, 30-56 (1987)

12. Gordon, A.Ya.: Pure point spectrum under 1-parameter perturbations and instability of Anderson localization. Commun. Math. Phys. 164, 489-505 (1994)

13. Gredeskul, S.A. and Pastur, L.A.: Behavior of the density of states in one-dimensional disordered systems near the edges of the spectrum. Theor. Math. Phys. 23, 132-139 (1975)

14. Hardy, G.H. and Wright, E.M.: An Introduction to the Theory of Numbers, 5th ed., Oxford: Oxford University Press, 1979

15. Harris W.A. and Lutz, D.A.: Asymptotic integration of adiabatic oscillator. J. Math. Anal. Appl. 51, 76-93 (1975)

16. Jitomirskaya, S. and Last, Y.: Dimensional Hausdorff properties of singular continuous spectra. Phys. Rev. Lett. 76, 1765-1769 (1996)

17. Jitomirskaya, S. and Last, Y.: Power law subordinacy and singular spectra I. Half-line operators. In preparation

18. Kahane, J.P.: Some Random Series of Functions. Cambridge: Cambridge University Press, 1985

19. Kiselev, A.A.: Absolutely continuous spectrum of one-dimensional Schrödinger operators and Jacobi matrices with slowly decreasing potentials. Commun. Math. Phys. 179, 377-400 (1996)

20. Kiselev, A., Remling, C. and Simon, B.: Effective perturbation methods for one-dimensional Schrödinger operators. Preprint

21. Kotani, S. and Ushiroya, N.: One-dimensional Schrödinger operators with random decaying potentials. Commun. Math. Phys. 115, 247-266 (1988)

22. Last, Y. and Simon, B.: Eigenfunctions, transfer matrices, and absolutely continuous spectrum of onedimensional Schrödinger operators. To appear in Invent. math.

23. Molchanov, S.: One-dimensional Schrödinger operators with sparse potentials. Preprint

24. Naboko, S.: Dense point spectra of Schrödinger and Dirac operators. Theor. Math. Phys. 68, 18-28 (1986)

25. Naboko, S. and Yakovlev, S.I.: On the point spectrum of discrete Schrödinger operators. Func. Anal. Appl 26, 85-88 (1992)

26. Pastur, L. and Figotin, A.: Spectra of Random and Almost-Periodic Operators. Berlin: Springer, 1992

27. Pearson, D.B.: Singular continuous measures in scattering theory. Commun. Math. Phys. 60, 13-36 (1978)

28. Pearson, D.B.: Pathological spectral properties. Mathematical Problems in Theoretical Physics, Proc. Internat. Conf. Math. Phys., Lausanne, Lecture Notes in Phys. 116, 1979, Berlin-New York: Springer, 1980, pp. 49-51

29. Remling, C.: A probabilistic approach to one-dimensional Schrödinger operators with sparse potentials. Commun. Math. Phys. 185, 313-323 (1997) 
30. Ruelle, D.: Ergodic theory of differentiable dynamical systems. Publ. Math. IHES 50, 275-306 (1979)

31. Simon, B.: Some Jacobi matrices with decaying potentials and dense point spectrum. Commun. Math. Phys. 87, 253-258 (1982)

32. Simon, B.: Spectral analysis and rank one perturbations and applications. CRM Lecture Notes Vol. 8, J. Feldman, R. Froese, L. Rosen, eds., Providence, RI: Amer. Math. Soc., 1995, pp. 109-149

33. Simon, B.: Bounded eigenfunctions and absolutely continuous spectra for onedimensional Schrödinger operators. Proc. Amer. Math. Soc. 124, 3361-3369 (1996)

34. Simon, B.: Some Schrödinger operators with dense point spectrum. Proc. Amer. Math. Soc. 125, 203-208 (1997)

35. Simon, B. and Stolz, G.: Operators with singular continuous spectrum, V. Sparse potentials. Proc. Amer. Math. Soc. 124, 2073-2080 (1996)

36. Stolz, G.: Bounded solutions and absolute continuity of Sturn-Liouville operators. J. Math. Anal. Appl. 169, 210-228 (1992)

37. Weidmann, J.: Zur Spektral theorie von Sturm-Liouville Operatoren. Math. Z. 98, 268-302 (1967)

Communicated by D. C. Brydges 